\title{
6. LAYER-BY-LAYER CORRELATION OF DRILLED SEDIMENTS FROM OWEN RIDGE, OMAN MARGIN, AND INDUS FAN 1
}

\author{
N. Niitsuma ${ }^{2}$
}

\begin{abstract}
One hundred thirty-one marker horizons relating to the distinct and traceable layers were described for the Owen Ridge and Oman Margin sites. The correlations incorporated the calculations of true depth, corrected for coring disturbance and gas expansion. Intersite correlation of marker horizons has been improved based on color density data, measured with video densitometer, and oxygen isotope stratigraphic data.

Distinct hiatuses were detected by the intersite correlation of the marker horizons in the Owen Ridge. The hiatuses are related to submarine slides induced by increasing gravitational instability for the accumulation of the pelagic sediments on the top of the Owen Ridge.

The large amount of sediment supply with variable lithofacies during the glacial stages is represented by layer-bylayer correlation in the Oman Margin.

The color density patterns with glacial-interglacial cycles are controlled by the balance of organic carbon content, increasing in the interglacial stages with strong upwelling induced by the southwest monsoon, and flux of terrigenous matter, increasing in the glacial stages. The present distinct climatic cycle relating to the southwest monsoon has been developed since Stage 8, $250 \mathrm{ka}$.

The large amount of sediment supply in the glacial stages can be assumed as fluvial in origin from the humid Arabian Peninsula, relating to the weakened Tropical Easterly Jet, which is induced by the counter-current of the southwest monsoon and maintains the present arid climate in the north Africa and Arabian Peninsula.
\end{abstract}

\section{INTRODUCTION}

Layer-by-layer correlation is a most fundamental method which is applied at the initial stage of the lithostratigraphic study just after the splitting a drilled core. Layer-by-layer correlation is most useful in making the interhole correlation necessary to yield a complete composite stratigraphic succession from the disturbed and partly missing sections resulting from drilling and whole round geochemical sampling.

The layer-by-layer correlation recognizes the marker horizons which can be traced over the studied area, giving the sequence of the lithofacies and lithostratigraphy. The lithologic sequence contains detailed records of environmental and sedimentational conditions, especially any sudden changes in lithofacies and bioturbation. The positioning of the sampling points within a detailed lithostratigraphical framework is important for discussions of environmental change based on measurements and/or analyses on samples taken routinely from cored sediments with arranged intervals. The positioning within a lithostratigraphical framework is absolutely needed for synthesis of the results of analyses and measurements, and to specify past phenomena.

Intersite correlation can be carried out by biostratigraphy, magnetostratigraphy, oxygen isotope stratigraphy, magnetic susceptibility pattern, color density pattern, and so on, however, the correlation is not detailed enough to specify which event produced various records in the sediments. Further detailed correlation can be realized by layer-by-layer correlation.

This paper gives the method of layer-by-layer correlation and shore-based studies, nomenclature system adopted in this work, description of the marker horizons in sediment cores taken from

\footnotetext{
${ }^{1}$ Prell, w. L., Niitsuma, N., et al., 1991. Proc. ODP, Sci. Results, 117: College Station, TX (Ocean Drilling Program).

2 Institute of Geosciences, Shizuoka University, 836 Oya, Shizuoka 422, Ja-
}

the Owen Ridge and Oman Margin, and discussion of the paleoclimatological and sedimentological phenomena in the northwestern Indian Ocean based on the layer-by-layer correlation.

\section{NOMENCLATURE SYSTEM OF MARKER HORIZONS}

For many of the sites drilled during Leg 117 in the northwestern Indian Ocean (Fig. 1), the dominant lithology consists of alternating layers of light, carbonate-rich intervals and darker, clay and organic-carbon-rich intervals. Interhole comparison of core photographs indicated that these sedimentary layers could be visibly correlated between holes based on their color, thickness, other characteristics, and their sequence. Using core photographs and whole-core magnetic susceptibility curves, the sedimentary layers could be correlated between holes to within 5$10 \mathrm{~cm}$. Several distinct and traceable layers were identified within the Owen Ridge sites and within the Oman Margin sites. One hundred thirty-one marker horizons relating to the distinct and traceable layers were described with the notation OR-A $A_{0}, A_{1}$, $A_{2}, \ldots, Z_{5}, Z_{6}$ for the Owen Ridge sites and $O M-a_{1}, a_{2}, \ldots, h_{4}$, $\mathrm{h}_{5}$ for the Oman Margin sites. The OR-A indicates that the marker horizon was first observed in Core 117-721A-1H of the first hole of the Owen Ridge sites, and the OR-B refers to horizons observed in Core 117-721A-2H. The numbered subscripts identify the horizon's sequence in a given core. Because the notation system was applied to both of the Owen Ridge sites and Oman Margin sites, prefix OR and capital letters are used for markers of the Owen Ridge sites, and prefix OM and small letters for the Oman Margin sites to avoid confusion. The prefixes have been already introduced in Prell, Niitsuma, et al. (1989).

\section{SHIPBOARD DETERMINATION OF MARKER HORIZONS}

Using black and white photographs of cores, marker horizons were examined. The photographs of the core were taken after cutting into $1.5 \mathrm{~m}$ sections, splitting in half, and recording the lithologic description. The quality of the photographs is 


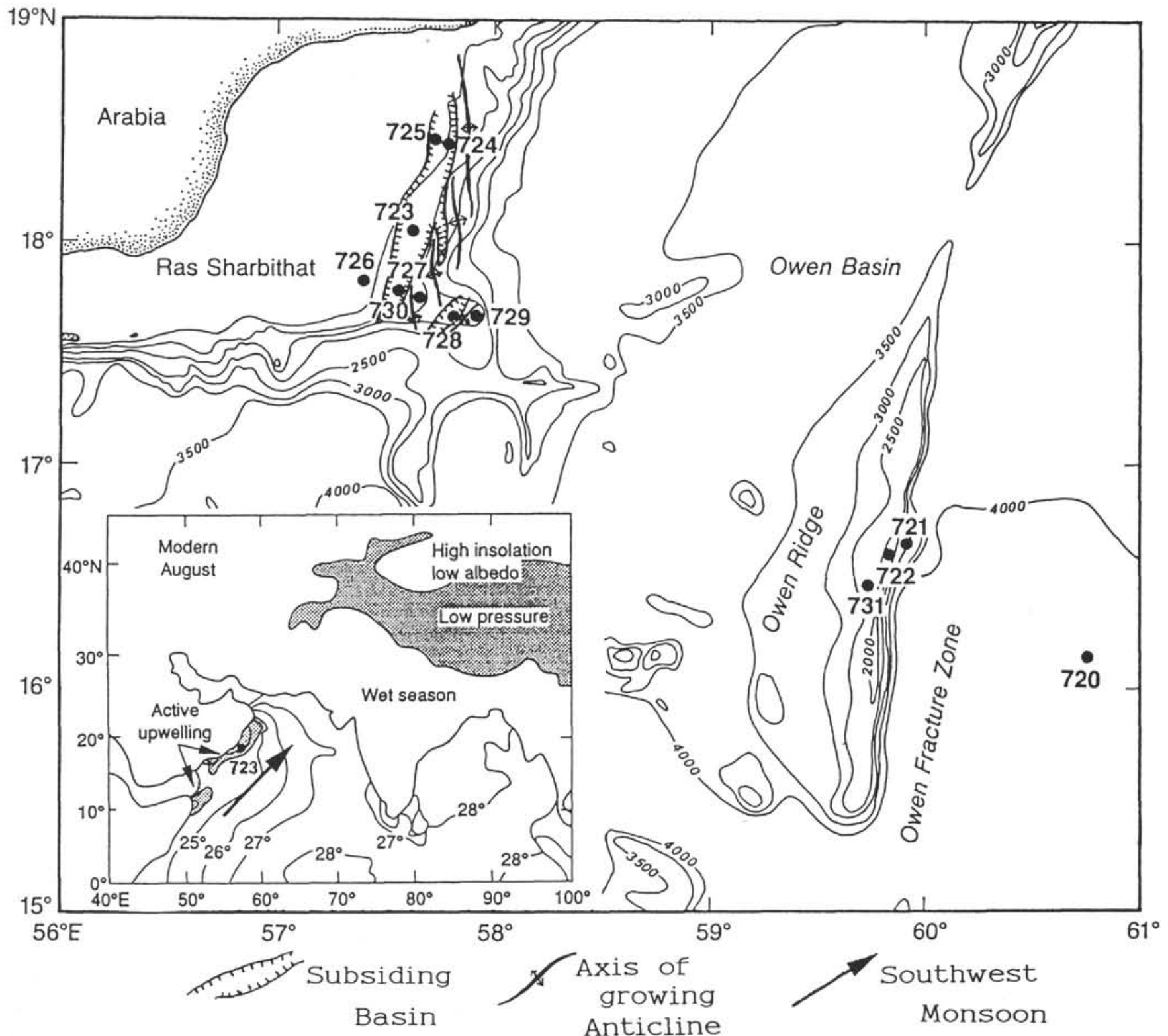

Figure 1. Location of ODP Leg 117 sites on the Owen Ridge, Oman Margin, and Indus Fan in the northwestern Indian Ocean. Tectonic setting of the Oman Margin sites and southwest monsoon are also shown.

good enough to recognize the lithologic character of the cored sediments. The core has missing intervals where whole round geochemical samples were taken, and the core top and bottom are always affected by the drilling disturbance and missing intervals.

The recognition procedures for the lithologic pattern and their correlation, using the photographs, were as following: reconstruct continuous lithologic sequence from cut pieces as sections; count the coring disturbance and missing intervals; recognize the lithologic pattern and layers which contain variation of color density, lamination, and bioturbation on each sedimentary sequence; and define and/or identify marker horizons selected at the clear and sharp boundary in the pattern without confusing and arbitrary identification for the correlation of the lithologic sequences.

Several marker horizons were usually defined in each core for continuous lithologic correlation during the comparison of the sequences from the other holes of the same site. Interhole correlations and calculation of the top depth of cores have been completed (Fig. 2).

Based on the layer-by-layer correlation referring to the marker horizons, intersite correlations were attempted. Magnetic susceptibility data were helpful in this effort. The intersite correlations were successful within the Owen Ridge sites, however, the correlations within the Oman Margin sites had uncertainties, especially with Sites 728 and 730 .

\section{METHOD AND RESULTS OF SHORE-BASED STUDY}

\section{Color Density Measurement}

The color density pattern was quantified by Luzex II video densitometer. The measurement was made on the black and white photographs of each section of drilled core. Five hundred 


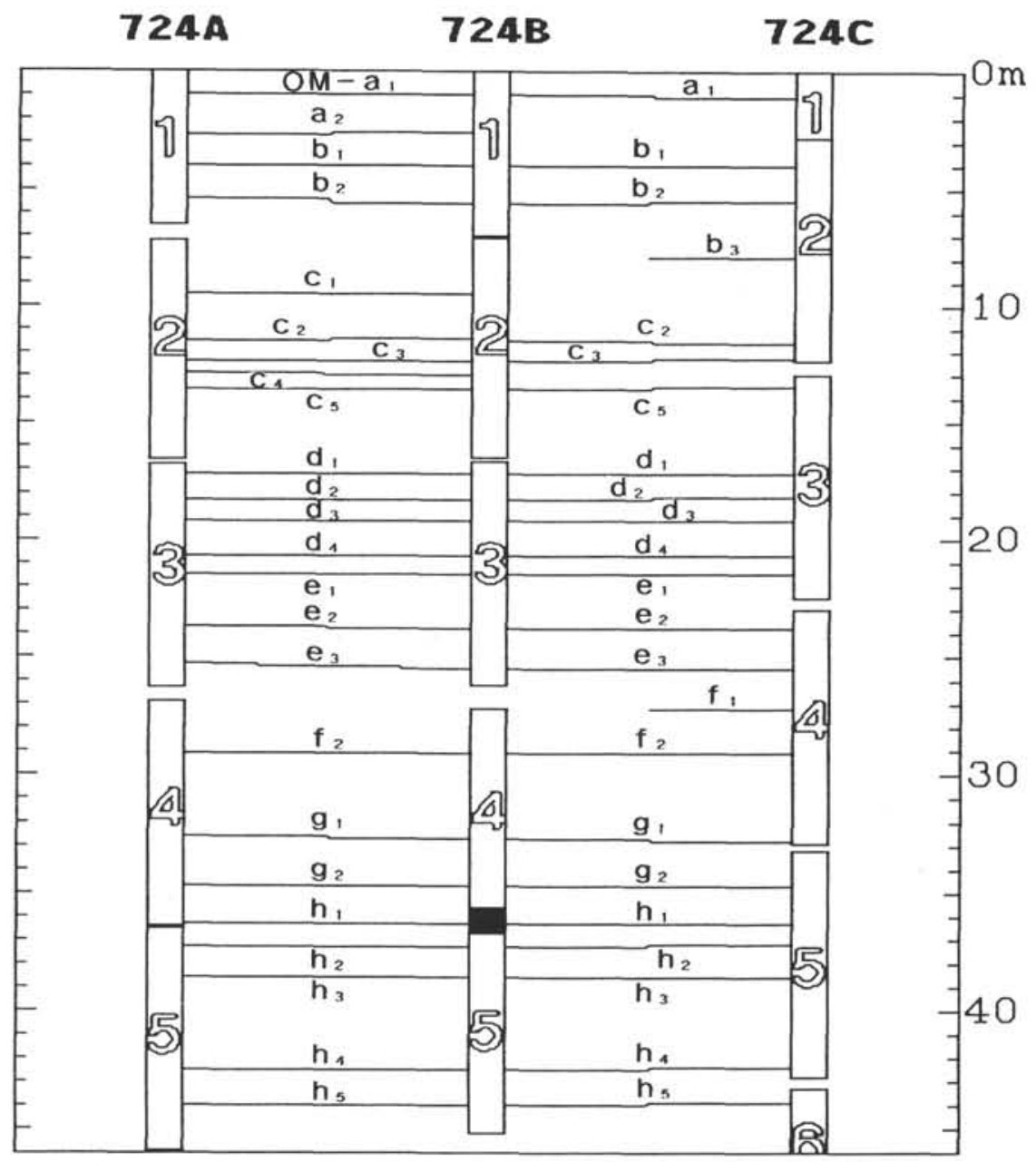

Figure 2. Example of interhole correlation of marker horizons and calculation of the top depth of cores in Site 724 on the Oman Margin.

averaged values for 12 measurements were obtained with 256 gradations along the core direction of the split surface of core section. The measured area corresponds to a $150 \mathrm{~cm} \times 3.6 \mathrm{~cm}$ rectangle at the axial part of a section of cored sediments. The resolution along the axis is $0.3 \mathrm{~cm}$ on the sediments. The gradations range from 30 for real black part to 220 for real white part on the photographs.

The effects of inhomogeneity of photograph illumination were detected in the measurement. Both ends of the section are lightest and its center is darkest for uniform color density section. The inhomogeneity can be approximated by a sine curve. The lightest points were calculated at $30 \mathrm{~cm}$ outside the ends of the section by fitting a sine curve. For analysis of the measurements, the inhomogeneity was subtracted numerically by fitting the sine curve.

The color density curves are useful to find a general trend of color pattern and to compare directly with magnetic susceptibility curves.

\section{Oxygen Isotope Stratigraphy}

Shore-based data of oxygen isotope stratigraphy are available for Sites 722 (Clemens and Prell, this volume), 723 (Niitsuma et al., this volume), 724 (Pedersen and Shimmield, this volume), and 728 (Kroon et al., this volume). The oxygen isotope stratigraphic correlation can be used not only for testing of the shipboard layer-by-layer correlation but also for positioning the marker horizons on oxygen isotopic stages and for regional correlation between the Owen Ridge sites and Oman Margin sites.

\section{DESCRIPTION OF MARKER HORIZONS}

\section{Owen Ridge Sites}

The type sections on which the marker horizon was originally defined follow:

OR-A $\mathrm{A}_{0}$ to $\mathrm{I}_{4}$ : Cores $117-721 \mathrm{~A}-1 \mathrm{H}$ to $9 \mathrm{H}$

OR- $\mathrm{J}_{1}$ to $\mathrm{Y}_{2}$ : : Cores $117-721 \mathrm{~B}-10 \mathrm{X}$ to $23 \mathrm{X}$

OR-K ${ }_{2}$ : Core 117-722A-11X

OR- $\mathrm{Z}_{1}$ to $\mathrm{Z}_{6}$ : Core $117-722 \mathrm{~B}-27 \mathrm{X}$ to $38 \mathrm{X}$

The descriptions of the marker horizons are shown in Table 1. The interhole correlation of marker horizons allows us to calculate true thickness between the horizons that coincide with and are separated by core boundaries. Occasionally core tops were expanded by water uptake during drilling. In this case, the apparent thickness of the disturbed sediment layer is observed to be thicker than the original thickness. In some instances, the top part of the core was missing; in this case apparent thickness is observed to be thinner than the original thickness. The origi- 
Table 1. Horizon and description of marker horizons for layer-by-layer correlation in the Owen Ridge (bioturbation; size in millimeters shown in parenthesis).

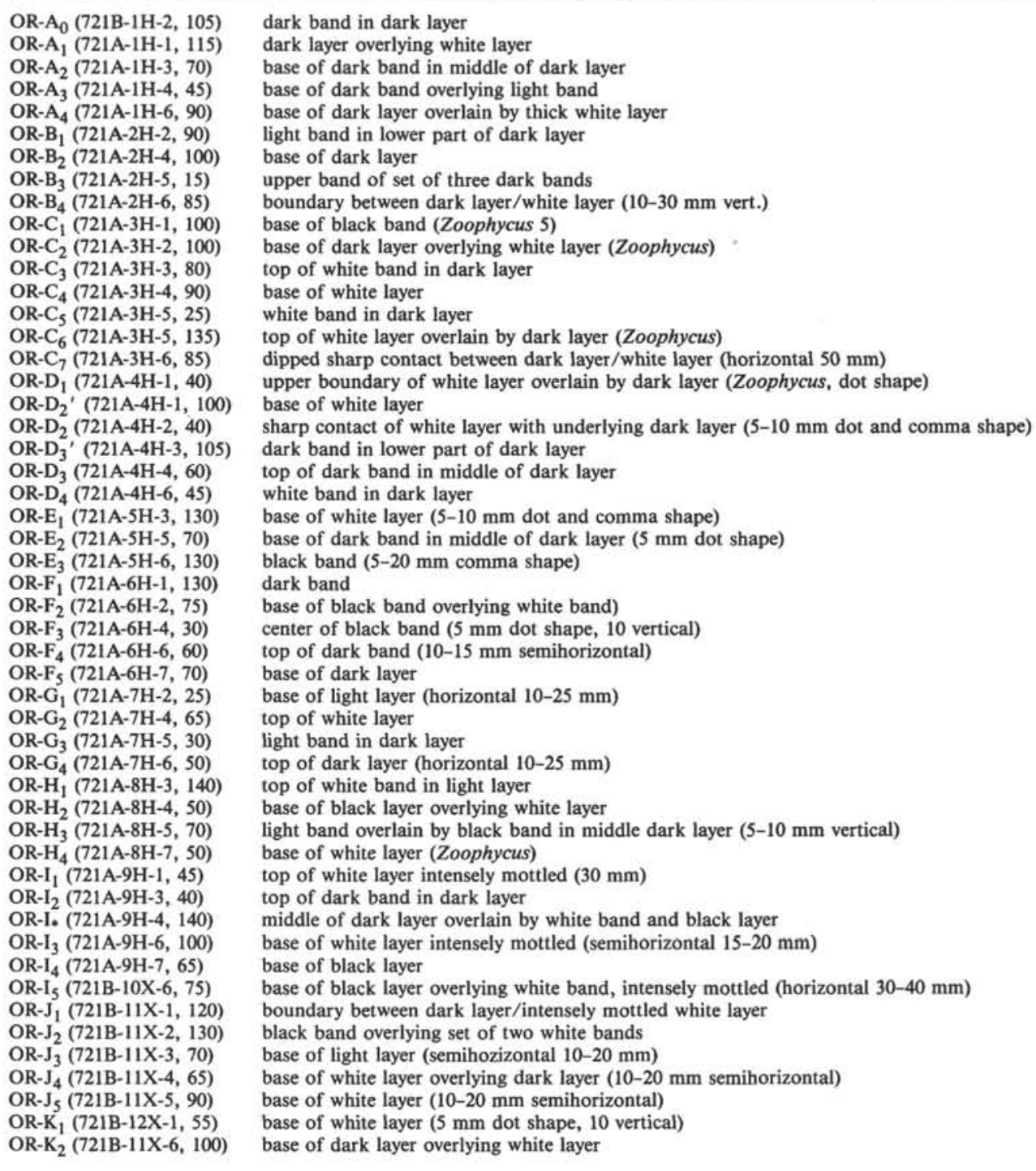

nal thickness between the marker horizons coinciding with core tops or bottoms can be obtained by comparing the thickness between the marker horizons in the continuous section of the other nearby holes. The horizons in the cored section and stratigraphic depth of the marker horizons in the Owen Ridge holes are given in Table 2.

The main framework of the intersite correlation in the Owen Ridge was reconstructed by using significant and traceable light layers below marker horizon OR- $A_{2}$, above $B_{1}$, below $B_{4}$, and below $C_{7}$, dark layer above $F_{1}$, light layer between $G_{4}$ and $H_{1}$, dark layer between $I_{2}$ and $I_{*}$, and light layers between $I_{5}$ and $J_{1}$, and light layers above $J_{4}$, above $M_{1}$, above $N_{2}$, above $R_{1}$, at $S_{4}$, below $U_{2}$, and below $X_{1}$. Because rhythmic changes in color density were observed below marker horizon $L_{1}$, the positions of the node in the oscillated pattern were also used for the correlation, especially below marker horizon $\mathrm{Y}_{2}$.

Table 3 shows the corrected core top depths, based on the interhole correlation of the marker horizons. The stratigraphic depths of the marker horizons in Table 2 were calculated using the core top data.

\section{Oman Margin sites}

The type section on which the marker horizon was originally defined follow:

$$
\begin{aligned}
& \text { OM-a } a_{1} \text { to } h_{4}: \text { Core } 117-723 \mathrm{~A}-1 \mathrm{H} \text { to } 9 \mathrm{H} \\
& \text { OM-h }
\end{aligned}
$$

The descriptions of the marker horizons are given in Table 4. The horizons in the cored section and stratigraphic depth of the marker horizons in the Oman Margin holes are listed in Table 5. Gaseous sediments were recovered in Sites 723 and 724, and the marker horizons were pushed down by the gas expansion after the recovery. Table 5 also shows the cumulative amount of gas expansion from the core top, and the gas expansion is used in the calculation of the stratigraphic depths. Table 6 shows the corrected core top depths, based on the interhole correlation of the marker horizons. The stratigraphic depths of the marker horizons in Table 5 were calculated using the core top depth. 
Table 1 (continued).

\begin{tabular}{|c|c|}
\hline OR-L ${ }_{1}(721 \mathrm{~B}-13 \mathrm{X}-1,60)$ & intensely mottled with band overlying black layer \\
\hline OR-L $(721 \mathrm{~B}-13 \mathrm{X}-6,60)$ & top of white band in dark layer) \\
\hline OR- $\mathrm{L}_{3}(721 \mathrm{~B}-13 \mathrm{X}-4,40)$ & center of white band in middle of dark layer \\
\hline OR- $\mathrm{L}_{4}(721 \mathrm{~B}-13 \mathrm{X}-6,30)$ & top of black layer \\
\hline OR-M $M_{1}(721 B-14 X-2,115)$ & base of light layer \\
\hline OR-M $M_{2}(721 B-14 X-3,120)$ & base of white layer overlying black layer \\
\hline OR-M $M_{3}(721 \mathrm{~B}-14 \mathrm{X}-4,70)$ & center of dark layer \\
\hline OR-N $N_{1}(721 B-15 X-1,75)$ & white band in light layer \\
\hline OR-N $2(721 \mathrm{~B}-15 \mathrm{X}-2,135)$ & top of white layer \\
\hline OR-N $3(721 \mathrm{~B}-15 \mathrm{X}-3,130)$ & center of white layer \\
\hline OR-N $N_{4}(721 \mathrm{~B}-15 \mathrm{X}-6,15)$ & top of dark layer \\
\hline OR-O $\mathrm{O}_{1}(721 \mathrm{~B}-16 \mathrm{X}-2,70)$ & base of light band in dark layer \\
\hline OR-O ${ }_{2}(721 \mathrm{~B}-16 \mathrm{X}-3,30)$ & base of white layer overlying black layer $(10-20 \mathrm{~mm}$ vertical) \\
\hline OR-P $P_{1}(721 \mathrm{~B}-17 \mathrm{X}-1,95)$ & base of white layer \\
\hline OR-P ${ }_{2}(721 \mathrm{~B}-17 \mathrm{X}-2,45)$ & base of white layer \\
\hline OR-P ${ }_{3}(721 \mathrm{~B}-17 \mathrm{X}-3,90)$ & top of white layer \\
\hline OR-P $P_{4}(721 \mathrm{~B}-17 \mathrm{X}-4,70)$ & base of white layer ( $10 \mathrm{~mm}$ egg shape) \\
\hline OR-P 5 (721B-17X-5, 100) & base of white layer \\
\hline OR-P $6(721 B-17 X-6,90)$ & base of top dark band in dark layer \\
\hline OR-Q $Q_{1}(721 \mathrm{~B}-18 \mathrm{X}-1,120)$ & base of white layer ( $5 \mathrm{~mm}$ vertical) \\
\hline OR-Q $2(721 B-18 X-2,90)$ & base of white layer overlying black layer (5-20 mm vertical) \\
\hline OR-Q $3(721 \mathrm{~B}-18 \mathrm{X}-3,45)$ & base of white band in dark layer \\
\hline OR-Q 4 (721B-18X-4, 100) & base of black layer overlying white band \\
\hline OR-Q $Q_{5}(721 \mathrm{~B}-18 \mathrm{X}-5,70)$ & base of dark layer overlying white layer \\
\hline OR-Q 6 (721B-18X-6, 90) & base of white layer overlying dark layer (horizontal $20 \mathrm{~mm}$ ) \\
\hline OR-R $R_{1}(721 \mathrm{~B}-19 \mathrm{X}-1,110)$ & base of white layer overlying black layer ( $5 \mathrm{~mm}$ dot shape, $20 \mathrm{~mm}$ horizontal) \\
\hline OR-R $(721 B-19 X-2,55)$ & base of white layer overlying black layer ( $5 \mathrm{~mm}$ dot shape, $20 \mathrm{~mm}$ horizontal) \\
\hline OR-R $3(721 \mathrm{~B}-19 \mathrm{X}-4,70)$ & base of white layer overlying dark layer ( $5 \mathrm{~mm}$ dot shape) \\
\hline$O R-R_{4}(721 B-19 X-5,100)$ & top of white layer overlain by dark layer \\
\hline OR-S ${ }_{1}(721 \mathrm{~B}-20 \mathrm{X}-2,105)$ & base of white layer overlying dark layer (20-30 mm vertical) \\
\hline OR-S $2(721 \mathrm{~B}-20 \mathrm{X}-3,100)$ & top of white layer overlain by dark layer \\
\hline OR-S 3 (721B-20X-4, 65) & base of white layer overlying dark layer (20-30 mm horizontal) \\
\hline $\mathrm{OR}-\mathrm{S}_{4}(721 \mathrm{~B}-20 \mathrm{X}-4,140)$ & center of white layer \\
\hline OR-T $(721 \mathrm{~B}-21 \mathrm{X}-1,20)$ & base of white layer overlying black layer (10-15 mm dot shape) \\
\hline OR-T $2(721 B-21 X-2,110)$ & base of white band in midle of light layer \\
\hline OR-U $\mathrm{U}_{1}(721 \mathrm{~B}-22 \mathrm{X}-3,30)$ & base of dark layer overlying white layer \\
\hline OR-U $2(721 \mathrm{~B}-22 \times 4,65)$ & base of dark layer overlying white layer \\
\hline OR-V $(721 \mathrm{~B}-23 \mathrm{X}-2,45)$ & boundary between dark layer/white layer \\
\hline OR-V $(721 \mathrm{~B}-23 \mathrm{X}-3,120)$ & bioturbated black band at boundary between dark layer/white layer \\
\hline $\mathrm{OR}_{1}(721 \mathrm{~B}-24 \mathrm{X}-2,60)$ & boundary of dark layer/light layer \\
\hline OR-W 2 (721B-24X-3, 100) & middle of dark layer \\
\hline OR-X $\mathrm{X}_{1}(721 \mathrm{~B}-25 \mathrm{X}-3,125)$ & middle of dark layer \\
\hline OR-X $2(721 \mathrm{~B}-25 \mathrm{X}-5,65)$ & base of dark layer overlying white layer \\
\hline OR-Y, $(721 \mathrm{~B}-26 \mathrm{X}-3,50)$ & base of dark layer overlying white layer \\
\hline OR-Y $2(721 \mathrm{~B}-26 \mathrm{X}-4,90)$ & dark part in dark layer (2-5 mm dot) \\
\hline OR-Z $Z_{1}(721 B-27 X-2,120)$ & black band in middle of dark layer at top of rhythmic alternation of dark and white layers (15 mm horizontal) \\
\hline OR-Z 2 (721B-29X-1, 110) & base of white layer intercalated by black layers ( $3 \mathrm{~mm}$ dot shape, $10 \mathrm{~mm}$ horizontal) \\
\hline $\mathrm{OR}-\mathrm{Z}_{3}(721 \mathrm{~B}-31 \mathrm{X}-2,120)$ & very white layer overlying dark layer which is intensely bioturbated by Chondrites (1-2 mm Chondrites, $10 \mathrm{~mm}$ horizontal) \\
\hline $\mathrm{OR}-\mathrm{Z}_{4}(721 \mathrm{~B}-35 \mathrm{X}-4,55)$ & lower band of three sets of dark bands in white layer (5-10 mm horizontal) \\
\hline $\mathrm{OR}-\mathrm{Z}_{5}(721 \mathrm{~B}-37 \mathrm{X}-3,40)$ & center of black layer ( $1 \mathrm{~mm}$ Chondrites in upper part, $20 \mathrm{~mm}$ circular with purple rings in middle) \\
\hline OR-Z $6(721 B-38 X-4,65)$ & black layer (1-5 mm horizontal, $15 \mathrm{~mm}$ circular with purple rings) \\
\hline
\end{tabular}

The main framework of the intersite correlation in the Oman Margin was reconstructed by using two significant and traceable light layers above marker horizon $O M-f_{1}$ and above $g_{1}$. The shallowest light layer below $a_{1}$, and light layers below $b_{2}$, below $\mathrm{c}_{1}$ and below $\mathrm{e}_{1}$ assisted and completed the intersite correlation.

\section{REEXAMINATION OF SHIPBOARD CORRELATION}

The intersite correlation of the marker horizons, mentioned above, is a result of reexamination of shipboard correlation (Prell, Niitsuma, et al., 1989), based on color density data and oxygen isotope stratigraphic data.

The correlation of OR- $A_{4}$ and $C_{1}-E_{2}$ of Site 731 with Sites 721 and 722 of the Owen Ridge has been improved based on color density data. The mismatching in the shipboard correlation was caused by missing intervals at the core breaks in Site 731 , where correlative sedimentary sequence was recovered only from Hole 731A.

The correlation of OM- $a_{2}, b_{1}$, and $c_{4}$ of Site 727 with Sites 723 and 724 of the Oman Margin has been improved to realize the better matching on the color density data.

The correlation of the upper half of marker horizons, OM-a to $e_{2}$ and $f_{1}$, of Sites 728 and 730 with that of Sites 723,724 , and
727 in the Oman Margin has been readjusted based on oxygen isotope stratigraphic data. The mismatching in the shipboard correlations resulted from the considerable difference and changes in the rate of sedimentation through the drilled sequences; Sites 728 and 730 have two to four times smaller sedimentation rate than Sites 723 and 727; Site 724 has a much higher rate within the interval from $c_{2}$ to $d_{4}$. In the shipboard correlation, light layer below $b_{2}$ was mismatched with light layer below $b_{1}$; light layer below $c_{1}$ was mismatched with light layer below $b_{3}$; light layer below $e_{1}$ which was not clear in Sites 724 and 727 was mismatched with light interval from $c_{5}$ to $d_{1}$.

\section{RELATIVE RATE OF SEDIMENTATION}

The marker horizons which can be traced in the sedimentary sequences of the Owen Ridge and Oman Margin are useful tools to determine the existence of hiatus and spatial changes in the rate of sedimentation by their relative rate of sedimentation.

\section{Hiatus in the Sedimentary Sequences on the Owen Ridge}

The pelagic sediments covering the Owen Ridge are uniform sedimentary conditions at Sites 721,722 , and 731 with sedimentation rates of $35-48 \mathrm{~m} / \mathrm{m}$.y. (Prell, Niitsuma, et al., 1989). The 
Table 2. Depth of marker horizons in the Owen Ridge holes.

\begin{tabular}{|c|c|c|c|c|c|c|c|c|c|c|c|c|}
\hline $\begin{array}{l}\text { Marker } \\
\text { horizon }\end{array}$ & $\begin{array}{c}\text { Hole } 721 \mathrm{~A} \\
\text { Core, section, } \\
\text { interval }(\mathrm{cm})\end{array}$ & $\begin{array}{l}\text { Depth } \\
\text { (mbsf) }\end{array}$ & $\begin{array}{l}\text { Hole 721B } \\
\text { Core, section, } \\
\text { interval }(\mathrm{cm})\end{array}$ & $\begin{array}{l}\text { Depth } \\
\text { (mbsf) }\end{array}$ & $\begin{array}{l}\text { Hole } 721 \mathrm{C} \\
\text { Core, section, } \\
\text { interval }(\mathrm{cm})\end{array}$ & $\begin{array}{l}\text { Depth } \\
\text { (mbsf) }\end{array}$ & $\begin{array}{l}\text { Hole } 722 \mathrm{~A} \\
\text { Core, section, } \\
\text { interval }(\mathrm{cm})\end{array}$ & $\begin{array}{l}\text { Depth } \\
\text { (mbsf) }\end{array}$ & $\begin{array}{l}\text { Hole 722B } \\
\text { Core, section, } \\
\text { interval }(\mathrm{cm})\end{array}$ & $\begin{array}{l}\text { Depth } \\
\text { (mbsf) }\end{array}$ & $\begin{array}{c}\text { Hole } 731 \mathrm{~A} \\
\text { Core, section, } \\
\text { interval }(\mathrm{cm})\end{array}$ & $\begin{array}{l}\text { Depth } \\
\text { (mbsf) }\end{array}$ \\
\hline OR-A $A_{2}$ & & & $1 \mathrm{H}-2,105$ & 2.55 & $1 \mathrm{H}-2,95$ & 2.55 & $1 \mathrm{H}-1,50$ & 2.3 & $1 \mathrm{H}-2,85$ & 2.35 & $1 \mathrm{H}-1,75$ & 0.75 \\
\hline$A_{1}$ & $1 \mathrm{H}-1,115$ & 3.95 & $1 \mathrm{H}-3,90$ & 3.9 & & & $1 \mathrm{H}-2,35$ & 3.65 & $1 \mathrm{H}-3,50$ & 3.5 & $1 \mathrm{H}-2,75$ & 2.25 \\
\hline$A_{2}$ & $1 \mathrm{H}-3,70$ & 6.5 & $1 \mathrm{H}-5,45$ & 6.45 & $2 \mathrm{H}-2,95$ & 6.55 & $1 \mathrm{H}-3,135$ & 6.15 & $2 \mathrm{H}-1,25$ & 6.05 & $1 \mathrm{H}-4,15$ & 4.65 \\
\hline $\mathrm{A}_{3}$ & $1 \mathrm{H}-4,45$ & 7.75 & $1 \mathrm{H}-6,32$ & 7.82 & $2 \mathrm{H}-3,65$ & 7.75 & $1 \mathrm{H}-4,115$ & 7.45 & $2 \mathrm{H}-2,20$ & 7.5 & $1 \mathrm{H}-5,45$ & 6.45 \\
\hline$A_{4}$ & $1 \mathrm{H}-6,90$ & 11.2 & $2 \mathrm{H}-2,25$ & 11.15 & $2 \mathrm{H}-5,105$ & 11.15 & $1 \mathrm{H}-7,30$ & 11.1 & $2 \mathrm{H}-4,65$ & 10.95 & & \\
\hline $\mathrm{B}_{1}$ & $2 \mathrm{H}-2,90$ & 15.1 & $2 \mathrm{H}-4,135$ & 15.25 & $3 \mathrm{H}-1,100$ & 15.2 & $2 \mathrm{H}-3,20$ & 14.2 & & & $2 \mathrm{H}-4,55$ & 14.85 \\
\hline$B_{2}$ & $2 \mathrm{H}-4,100$ & 18.2 & $2 \mathrm{H}-6,115$ & 18.05 & $3 \mathrm{H}-3,95$ & 18.15 & $2 \mathrm{H}-5,95$ & 17.95 & $3 \mathrm{H}-2,105$ & 17.95 & $2 \mathrm{H}-6,120$ & 18.5 \\
\hline$B_{3}$ & $2 \mathrm{H}-5,15$ & 18.85 & $2 \mathrm{H}-7,40$ & 18.8 & $3 \mathrm{H}-4,20$ & 18.9 & $2 \mathrm{H}-6,30$ & 18.8 & $3 \mathrm{H}-3,45$ & 18.85 & $2 \mathrm{H}-7,45$ & 19.25 \\
\hline $\mathrm{B}_{4}$ & $2 \mathrm{H}-6,85$ & 21.05 & $3 \mathrm{H}-1,140$ & 21 & $3 \mathrm{H}-5,100$ & 21.2 & $3 \mathrm{H}-1,60$ & 21.4 & $3 \mathrm{H}-5,10$ & 21.5 & $3 \mathrm{H}-2,85$ & 21.65 \\
\hline$C_{1}$ & $3 \mathrm{H}-1,100$ & 23.9 & $3 \mathrm{H}-3,100$ & 23.6 & & & $3 \mathrm{H}-3,85$ & 24.65 & $3 \mathrm{H}-7,15$ & 24.55 & $3 \mathrm{H}-4,90$ & 24.7 \\
\hline $\mathrm{C}_{2}$ & $3 \mathrm{H}-2,100$ & 25.4 & $3 \mathrm{H}-4,130$ & 25.4 & $4 \mathrm{H}-1,140$ & 25.5 & $3 \mathrm{H}-4,115$ & 26.45 & $4 \mathrm{H}-1,85$ & 26.35 & $3 \mathrm{H}-5,55$ & 25.85 \\
\hline $\mathrm{C}_{3}$ & $3 \mathrm{H}-3,80$ & 26.7 & $3 \mathrm{H}-5,105$ & 26.65 & $4 \mathrm{H}-2,125$ & 26.85 & $3 \mathrm{H}-5,85$ & 27.65 & $4 \mathrm{H}-2,60$ & 27.6 & $3 \mathrm{H}-5,145$ & 26.75 \\
\hline $\mathrm{C}_{4}$ & $3 \mathrm{H}-4,90$ & 28.3 & $3 \mathrm{H}-6,110$ & 28.2 & $4 \mathrm{H}-3,145$ & 28.55 & $3 \mathrm{H}-6,100$ & 29.3 & $4 \mathrm{H}-3,85$ & 29.35 & $3 \mathrm{H}-7,55$ & 28.85 \\
\hline $\mathrm{C}_{5}$ & $3 \mathrm{H}-5,25$ & 29.15 & $3 \mathrm{H}-7,50$ & 29.1 & $4 \mathrm{H}-4,55$ & 29.15 & $3 \mathrm{H}-7,40$ & 30.2 & $4 \mathrm{H}-4,40$ & 30.4 & $4 \mathrm{H}-1,125$ & 30.05 \\
\hline $\mathrm{C}_{6}$ & $3 \mathrm{H}-5,135$ & 30.25 & $4 \mathrm{H}-1,75$ & 30.25 & $4 \mathrm{H}-5,35$ & 30.45 & $4 \mathrm{H}-1,30$ & 31.4 & $4 \mathrm{H}-5,5$ & 31.55 & $4 \mathrm{H}-2,120$ & 31.5 \\
\hline $\mathrm{C}_{7}$ & $3 \mathrm{H}-6,85$ & 31.25 & $4 \mathrm{H}-2,20$ & 31.2 & $4 \mathrm{H}-5,120$ & 31.3 & $4 \mathrm{H}-1,140$ & 32.5 & $4 \mathrm{H}-5,100$ & 32.5 & $4 \mathrm{H}-3,55$ & 32.35 \\
\hline $\mathrm{D}_{1}^{\prime}$ & & & & & & & $4 \mathrm{H}-3,65$ & 34.75 & $4 \mathrm{H}-6,140$ & 34.4 & $4 \mathrm{H}-4,100$ & 34.3 \\
\hline $\mathrm{D}_{1}$ & $4 \mathrm{H}-1,40$ & 34 & $4 \mathrm{H}-4,0$ & 34 & & & $4 \mathrm{H}-4,5$ & 35.65 & & & $4 \mathrm{H}-5,55$ & 35.35 \\
\hline $\mathrm{D}_{2}$ & $4 \mathrm{H}-1,100$ & 34.6 & $4 \mathrm{H}-4,70$ & 34.7 & $5 \mathrm{H}-1,50$ & 34.8 & $4 \mathrm{H}-4,65$ & 36.25 & $5 \mathrm{H}-1,70$ & 36 & $4 \mathrm{H}-5,85$ & 35.65 \\
\hline$D_{2}$ & $4 \mathrm{H}-2,40$ & 35.5 & $4 \mathrm{H}-4,145$ & 35.45 & $5 \mathrm{H}-1,130$ & 35.6 & $4 \mathrm{H}-4,150$ & 37.1 & $5 \mathrm{H}-2,10$ & 36.9 & $4 \mathrm{H}-6,25$ & 36.55 \\
\hline $\mathrm{D}_{3}$ & $4 \mathrm{H}-3,105$ & 37.65 & $4 \mathrm{H}-6,85$ & 37.85 & $5 \mathrm{H}-3,70$ & 38 & $4 \mathrm{H}-6,80$ & 39.4 & $5 \mathrm{H}-3,100$ & 39.3 & $5 \mathrm{H}-2,100$ & 40.8 \\
\hline$D_{3}$ & $4 \mathrm{H}-4,60$ & 38.7 & $4 \mathrm{H}-7,30$ & 38.8 & $5 \mathrm{H}-4,0$ & 38.8 & $4 \mathrm{H}-7,25$ & 40.35 & $5 \mathrm{H}-4,30$ & 40.1 & $5 \mathrm{H}-3,75$ & 42.05 \\
\hline $\mathrm{D}_{4}$ & $4 \mathrm{H}-6,45$ & 41.55 & $5 \mathrm{H}-1,125$ & 41.35 & $5 \mathrm{H}-5,105$ & 41.35 & $5 \mathrm{H}-1,90$ & 41.5 & $5 \mathrm{H}-5,20$ & 41.5 & $5 \mathrm{H}-5,135$ & 45.65 \\
\hline $\mathrm{E}_{1}$ & $5 \mathrm{H}-3,130$ & 48.3 & $5 \mathrm{H}-6,75$ & 48.35 & $6 \mathrm{H}-3,110$ & 48.3 & $5 \mathrm{H}-6,115$ & 49.25 & $6 \mathrm{H}-3,130$ & 49.3 & $6 \mathrm{H}-6,105$ & 56.35 \\
\hline$E_{2}$ & $5 \mathrm{H}-5,70$ & 50.7 & $6 \mathrm{H}-1,35$ & 50.45 & $6 \mathrm{H}-5,60$ & 50.8 & $6 \mathrm{H}-1,105$ & 51.55 & $6 \mathrm{H}-5,60$ & 51.6 & $7 \mathrm{H}-2,130$ & 60.1 \\
\hline$E_{3}$ & $5 \mathrm{H}-6,130$ & 52.8 & $6 \mathrm{H}-2,130$ & 52.9 & $6 \mathrm{H}-6,130$ & 53 & $6 \mathrm{H}-3,40$ & 53.9 & $6 \mathrm{H}-6,130$ & 53.8 & $7 \mathrm{H}-4,135$ & 63.15 \\
\hline$F_{1}$ & $6 \mathrm{H}-1,130$ & 55.7 & $6 \mathrm{H}-4,115$ & 55.75 & $7 \mathrm{H}-2,15$ & 55.75 & $6 \mathrm{H}-4,120$ & 56.2 & $7 \mathrm{H}-1,45$ & 56.05 & $8 X-1,95$ & 67.95 \\
\hline$F_{2}$ & $6 \mathrm{H}-2,75$ & 56.65 & $6 \mathrm{H}-5,60$ & 56.7 & $7 \mathrm{H}-2,110$ & 56.7 & $6 \mathrm{H}-5,55$ & 57.05 & $7 \mathrm{H}-1,140$ & 57 & $8 \mathrm{X}-2,120$ & 69.7 \\
\hline $\mathrm{F}_{3}$ & $6 \mathrm{H}-4,30$ & 59.2 & $6 \mathrm{H}-7,15$ & 59.25 & $7 \mathrm{H}-4,80$ & 59.4 & $6 \mathrm{H}-6,130$ & 59.3 & $7 \mathrm{H}-3,85$ & 59.45 & $8 X-4,100$ & 72.5 \\
\hline $\mathrm{F}_{4}$ & $6 \mathrm{H}-6,60$ & 62.5 & $7 \mathrm{H}-3,45$ & 62.55 & $7 \mathrm{H}-6,100$ & 62.6 & $7 \mathrm{H}-2,45$ & 62.75 & $7 \mathrm{H}-5,100$ & 62.6 & $9 \mathrm{X}-2,80$ & 79 \\
\hline $\mathrm{F}_{5}$ & $6 \mathrm{H}-7,70$ & 64.1 & $7 \mathrm{H}-4,65$ & 64.25 & & & $7 \mathrm{H}-3,50$ & 64.3 & $7 \mathrm{H}-6,120$ & 64.3 & $9 X-3,90$ & 80.6 \\
\hline $\mathrm{G}_{1}$ & $7 \mathrm{H}-2,25$ & 65.55 & $7 \mathrm{H}-5,45$ & 65.55 & $8 \mathrm{H}-1,130$ & 65.6 & $7 \mathrm{H}-4,40$ & 65.7 & & & $9 X-4,85$ & 82.05 \\
\hline $\mathrm{G}_{2}$ & $7 \mathrm{H}-4,65$ & 68.95 & $8 \mathrm{H}-1,85$ & 69.05 & $8 \mathrm{H}-4,25$ & 69.05 & $7 \mathrm{H}-6,90$ & 69.2 & $8 \mathrm{H}-3,75$ & 69.25 & $10 X-1,5$ & 86.45 \\
\hline $\mathrm{G}_{3}$ & $7 \mathrm{H}-5,30$ & 70.1 & $8 \mathrm{H}-2,50$ & 70.2 & $8 \mathrm{H}-4,140$ & 70.2 & $8 \mathrm{H}-1,30$ & 71.2 & $8 \mathrm{H}-4,120$ & 71.2 & $10 \mathrm{X}-2,20$ & 88.1 \\
\hline $\mathrm{G}_{4}$ & $7 \mathrm{H}-6,50$ & 71.8 & $8 \mathrm{H}-3,70$ & 71.9 & $8 \mathrm{H}-6,10$ & 71.9 & $8 \mathrm{H}-2,45$ & 72.85 & $8 \mathrm{H}-5,140$ & 72.9 & $10 \mathrm{X}-3,15$ & 89.55 \\
\hline $\mathrm{H}_{1}$ & $8 \mathrm{H}-3,140$ & 77.7 & $8 \mathrm{H}-7,50$ & 77.7 & $9 \mathrm{H}-4,105$ & 77.65 & $8 \mathrm{H}-6,60$ & 79 & $9 \mathrm{H}-3,50$ & 79 & $11 X-1,5$ & 96.05 \\
\hline $\mathrm{H}_{2}$ & $8 \mathrm{H}-4,50$ & 78.3 & $9 \mathrm{H}-1,35$ & 78.25 & $9 \mathrm{H}-5,15$ & 78.25 & $8 \mathrm{H}-6,110$ & 79.5 & $9 \mathrm{H}-3,110$ & 79.6 & & \\
\hline $\mathrm{H}_{3}$ & $8 \mathrm{H}-5,70$ & 80 & $9 \mathrm{H}-2,60$ & 80 & $9 \mathrm{H}-6,25$ & 79.85 & & & $9 \mathrm{H}-4,110$ & 81.1 & & \\
\hline $\mathrm{H}_{4}$ & $8 \mathrm{H}-7,50$ & 82.8 & $9 \mathrm{H}-4,40$ & 82.8 & & & & & $9 \mathrm{H}-6,60$ & 83.6 & & \\
\hline$I_{1}$ & $9 \mathrm{H}-1,45$ & 84.35 & $9 \mathrm{H}-5,45$ & 84.35 & $10 \mathrm{H}-1,135$ & 84.45 & $9 \mathrm{H}-3,90$ & 85 & $9 \mathrm{H}-7,50$ & 85 & & \\
\hline$I_{2}$ & $9 \mathrm{H}-3,40$ & 87.3 & $9 \mathrm{H}-7,40$ & 87.3 & $10 \mathrm{H}-3,115$ & 87.25 & $9 \mathrm{H}-4,100$ & 86.6 & $10 \mathrm{H}-2,60$ & 86.4 & & \\
\hline I. & $9 \mathrm{H}-4,140$ & 89.8 & $10 X-2,50$ & 89.8 & $10 \mathrm{H}-5,70$ & 89.8 & $9 \mathrm{H}-5,70$ & 87.8 & $10 \mathrm{H}-3,75$ & 88.05 & & \\
\hline$I_{3}$ & $9 \mathrm{H}-6,100$ & 92.4 & $10 \times-4,25$ & 92.55 & $10 \mathrm{H}-7,60$ & 92.7 & $10 X-1,5$ & 90.75 & $10 \mathrm{H}-5,40$ & 90.7 & & \\
\hline$I_{4}$ & $9 \mathrm{H}-7,65$ & 93.55 & $10 X-4,120$ & 93.5 & $11 X-1,70$ & 93.5 & $10 \mathrm{X}-1,125$ & 91.95 & $10 \mathrm{H}-5,135$ & 91.65 & & \\
\hline 15 & & & $10 X-6,75$ & 96.05 & $11 X-3,40$ & 96.2 & $10 \mathrm{X}-3,25$ & 93.95 & & & & \\
\hline$J_{1}$ & & & $11 X-1,120$ & 98.2 & $11 X-4,90$ & 98.2 & $10 X-4,60$ & 95.8 & $11 X-2,5$ & 95.65 & & \\
\hline$J_{2}$ & & & $11 X-2,130$ & 99.8 & $11 X-5,100$ & 99.8 & $10 X-5,60$ & 97.3 & $11 X-3,35$ & 97.45 & & \\
\hline $\mathrm{J}_{3}$ & & & $11 X-3,70$ & 100.7 & $12 X-1,85$ & 100.55 & $10 \mathrm{X}-5,120$ & 97.9 & $11 X-3,80$ & 97.9 & & \\
\hline $\mathrm{J}_{4}$ & & & $11 X-4,65$ & 102.15 & $12 \mathrm{X}-2,90$ & 102.1 & $11 \mathrm{X}-1,50$ & 99.1 & $11 X-4,50$ & 99.1 & & \\
\hline $\mathrm{J}_{5}$ & & & $11 X-5,90$ & 103.9 & $12 \mathrm{X}-3,125$ & 103.95 & $11 X-2,95$ & 101.05 & & & & \\
\hline $\mathrm{K}_{1}$ & & & $12 \mathrm{X}-1,55$ & 105.45 & $12 X-4,130$ & 105.5 & $11 X-3,100$ & 102.6 & & & & \\
\hline $\mathrm{K}_{2}$ & & & & & & & $11 X-6,100$ & 107.1 & $12 X-3,45$ & 107.05 & & \\
\hline
\end{tabular}

lithofacies of the pelagic sediments are characterized by the cyclic alternation of lighter carbonate-rich layer and darker clayrich layer, however, some horizons of hiatus are determined by sharp contact of different layers and missing marker horizons. For the quantitative analyses of the hiatus and spatial difference of sedimentation rate, the relations of the depths of all correlative marker horizons in the Owen Ridge sites were examined and plotted on a graph (Fig. 3). All points representing the depth relation between the sites are directly connected by straight lines in the graph.

The depth relations of the marker horizons between Sites 721 and 722 gives a series of points arranged nearly on a straight line in the upper sequence shallower than marker horizon OR$\mathrm{N}_{2}$. Below OR- $\mathrm{N}_{2}$, the correlation line curves significantly, because the interval was covered by single Hole $721 \mathrm{C}$ in the site, and the core top depths could not be corrected for coring disturbances and incomplete recoveries. The difference clearly shows the importance of the interhole correlation for reconstruction of the stratigraphic succession.
The intersite correlation of the marker horizons between Sites 721 and 731 shows clearly the existence of a hiatus at Site 731 in the interval from OR- $\mathrm{H}_{1}$ to $\mathrm{N}_{1}$ (Fig. 3). The disconformable contact was observed at the horizon $75 \mathrm{~cm}$ below the marker horizon $\mathrm{OR}-\mathrm{H}_{1}$, and the marker horizon $\mathrm{OR}-\mathrm{N}_{1}$ was identified at $480 \mathrm{~cm}$ below the contact. The process of the identification of the marker horizon OR-N $\mathrm{N}_{1}$ follows: characteristic light layer below $O R-G_{1}$ is clearly identified above the hiatus; disturbed sedimentary structure is found within $2 \mathrm{~m}$ interval below the disconformable contact in Sections 731A-11X-1 and 11X-2; characteristic key layers, dark layer above OR-I., light layer below $\mathrm{I}_{5}$, and light layers above $\mathrm{J}_{4}$, and above $\mathrm{M}_{1}$ are missing below the disturbed interval; the sedimentary sequence below the disturbed interval is light color dominant more than the missing interval; and characteristic light layer above $\mathrm{N}_{1}$ is identified below the disturbed interval. The thickness between $\mathrm{H}_{1}$ and $\mathrm{N}_{1}$ is $49.75 \mathrm{~m}$ at Site 721, while the thickness at Site 731 is $5.55 \mathrm{~m}$.

Another sharp contact in Site 731 is observed $19.75 \mathrm{~m}$ below the previous hiatus, and marker OR- $\mathrm{X}_{1}$ was identified $2.10 \mathrm{~m}$ 
Table 2 (continued).

\begin{tabular}{|c|c|c|c|c|c|c|c|c|c|c|c|c|}
\hline $\begin{array}{l}\text { Marker } \\
\text { horizon }\end{array}$ & $\begin{array}{l}\text { Hole 721A } \\
\text { Core, section, } \\
\text { interval }(\mathrm{cm})\end{array}$ & $\begin{array}{l}\text { Depth } \\
\text { (mbsf) }\end{array}$ & $\begin{array}{l}\text { Hole } 721 \mathrm{~B} \\
\text { Core, section, } \\
\text { interval }(\mathrm{cm})\end{array}$ & $\begin{array}{l}\text { Depth } \\
\text { (mbsf) }\end{array}$ & $\begin{array}{l}\text { Hole } 721 \mathrm{C} \\
\text { Core, section, } \\
\text { interval }(\mathrm{cm})\end{array}$ & $\begin{array}{l}\text { Depth } \\
\text { (mbsf) }\end{array}$ & $\begin{array}{l}\text { Hole 722A } \\
\text { Core, section, } \\
\text { interval }(\mathrm{cm})\end{array}$ & $\begin{array}{l}\text { Depth } \\
\text { (mbsf) }\end{array}$ & $\begin{array}{l}\text { Hole 722B } \\
\text { Core, section, } \\
\text { interval }(\mathrm{cm})\end{array}$ & $\begin{array}{l}\text { Depth } \\
\text { (mbsf) }\end{array}$ & $\begin{array}{l}\text { Hole 731A } \\
\text { Core, section, } \\
\text { interval }(\mathrm{cm})\end{array}$ & $\begin{array}{l}\text { Depth } \\
\text { (mbsf) }\end{array}$ \\
\hline $\mathbf{L}_{1}$ & & & $13 X-1,60$ & 109.7 & $13 \mathrm{X}-1,90$ & 110 & $12 X-1,45$ & 109.95 & & & & \\
\hline $\mathbf{L}_{2}$ & & & $13 X-2,60$ & 111.2 & $13 \times-2,55$ & 111.15 & $12 X-2,45$ & 111.45 & & & & \\
\hline $\mathrm{L}_{3}$ & & & $13 X-4,40$ & 114 & $13 \times-4,15$ & 113.75 & $12 \times-4,10$ & 114.1 & $13 X-1,65$ & 113.95 & & \\
\hline $\mathrm{L}_{4}$ & & & $13 X-6,30$ & 116.9 & $14 X-2,45$ & 116.65 & $12 X-5,115$ & 116.65 & $13 X-3,45$ & 116.75 & & \\
\hline $\mathrm{M}_{1}$ & & & $14 X-2,115$ & 119.65 & $14 X-4,40$ & 119.6 & $13 \mathrm{X}-1,50$ & 119 & & & & \\
\hline $\mathrm{M}_{2}$ & & & $14 X-3,120$ & 121.2 & $14 X-5,55$ & 121.25 & $13 X-2,90$ & 120.9 & & & & \\
\hline $\mathrm{M}_{3}$ & & & $14 X-4,70$ & 122.2 & $15 X-1,60$ & 122.2 & $13 \mathrm{X}-3,35$ & 121.85 & & & & \\
\hline $\mathrm{N}_{1}$ & & & $15 X-1,75$ & 127.45 & $15 X-4,140$ & 127.5 & $13 X-6,95$ & 126.95 & $14 X-3,90$ & 126.9 & $11 X-4,110$ & 101.6 \\
\hline $\mathrm{N}_{2}$ & & & $15 X-2,135$ & 129.55 & $15 X-6,45$ & 129.55 & & & $14 X-4,65$ & 128.15 & $11 X-5,130$ & 103.3 \\
\hline $\mathrm{N}_{3}$ & & & $15 X-3,130$ & 131 & & & & & $14 X-5,20$ & 129.2 & $11 X-6,90$ & 104.4 \\
\hline $\mathrm{N}_{4}$ & & & $15 X-6,5$ & 134.25 & & & $14 X-3,45$ & 132.55 & $15 X-1,95$ & 133.55 & $12 \times-2,55$ & 107.75 \\
\hline $\mathrm{O}_{1}^{4}$ & & & $16 \times-2,70$ & 146 & & & $14 X-4,100$ & 134.6 & & & $12 \times-4,70$ & 110.9 \\
\hline $\mathrm{O}_{2}$ & & & $16 \times-3,30$ & 147.1 & & & $14 X-5,15$ & 135.25 & & & $12 X-5,10$ & 111.8 \\
\hline $\mathrm{P}_{1}^{2}$ & & & $17 X-1,95$ & 154.45 & & & $15 \times-2,120$ & 140.5 & & & & \\
\hline $\mathrm{P}_{2}$ & & & $17 X-2,45$ & 155.45 & & & $15 X-3,65$ & 141.45 & & & & \\
\hline $\mathrm{P}_{3}$ & & & $17 X-3,90$ & 157.4 & & & $15 \times-4,45$ & 142.75 & & & & \\
\hline $\mathrm{P}_{4}$ & & & $17 X-4,70$ & 158.7 & & & $15 X-5,40$ & 144.2 & $16 X-2,35$ & 144.15 & & \\
\hline$P_{5}$ & & & $17 X-5,100$ & 160.5 & & & $15 \times-6,70$ & 146 & $16 X-3,75$ & 146.05 & & \\
\hline$P_{6}$ & & & $17 X-6,90$ & 161.9 & & & $15 \times-7,20$ & 147 & $16 \times-4,15$ & 146.95 & & \\
\hline$Q_{1}^{0}$ & & & $18 X-1,120$ & 164.4 & & & $16 \times-1,65$ & 148.55 & $16 \times-5,35$ & 148.65 & & \\
\hline$Q_{2}$ & & & $18 X-2,90$ & 165.6 & & & $16 \times-2,30$ & 149.7 & $16 X-5,130$ & 149.6 & & \\
\hline$Q_{3}$ & & & $18 \times-3,45$ & 166.65 & & & $16 \times-2,110$ & 150.5 & $16 \times-6,60$ & 150.4 & & \\
\hline$Q_{4}$ & & & $18 X-4,100$ & 168.7 & & & $16 \mathrm{X}-3,100$ & 151.9 & & & & \\
\hline$Q_{5}$ & & & $18 X-5,70$ & 169.9 & & & $16 \times-4,30$ & 152.7 & & & & \\
\hline$Q_{6}$ & & & $18 X-6,90$ & 171.6 & & & $16 \times-5,15$ & 154.05 & $17 X-2,5$ & 154.05 & & \\
\hline$R_{1}$ & & & $19 X-1,110$ & 174 & & & $17 \mathrm{X}-1,125$ & 158.25 & $17 X-4,145$ & 158.45 & & \\
\hline $\mathrm{R}_{2}$ & & & $19 \times-2,55$ & 174.95 & & & $17 \times-2,110$ & 159.6 & $17 \times-5,75$ & 159.25 & & \\
\hline $\mathbf{R}_{3}$ & & & $19 \times-4,70$ & 178.1 & & & & & $17 \times-7,40$ & 161.9 & & \\
\hline $\mathbf{R}_{4}$ & & & $19 X-5,100$ & 179.9 & & & & & $18 \mathrm{X}-1,125$ & 163.35 & & \\
\hline $\mathrm{S}_{1}$ & & & $20 \times-2,105$ & 185.15 & & & & & $18 \times-4,130$ & 167.9 & & \\
\hline $\mathrm{S}_{2}$ & & & $20 \times-3,100$ & 186.6 & & & & & $18 X-6,50$ & 170.1 & & \\
\hline $\mathrm{S}_{3}$ & & & $20 X-4,65$ & 187.75 & & & & & $18 X-7,5$ & 171.15 & & \\
\hline $\mathrm{S}_{4}$ & & & $20 \times-4,140$ & 188.5 & & & $19 X-1,75$ & 177.05 & $19 \mathrm{X}-1,90$ & 176.8 & & \\
\hline$T_{1}$ & & & $21 X-1,20$ & 192.5 & & & $19 X-5,30$ & 182.6 & $19 X-5,90$ & 182.8 & & \\
\hline $\mathrm{T}_{2}$ & & & $21 X-2,110$ & 194.9 & & & $19 X-6,95$ & 184.75 & $20 \times-1,110$ & 184.8 & & \\
\hline $\mathrm{U}_{1}^{2}$ & & & $22 \times-3,30$ & 205.3 & & & $20 X-2,65$ & 188.15 & & & & \\
\hline $\mathrm{U}_{2}^{1}$ & & & $22 \times-4,65$ & 207.15 & & & $20 \mathrm{X}-3,75$ & 189.75 & & & & \\
\hline$v_{1}^{2}$ & & & $23 \mathrm{X}-2,45$ & 213.65 & & & & & $21 X-2,40$ & 195.2 & & \\
\hline $\mathrm{v}_{2}$ & & & $23 \mathrm{X}-3,120$ & 215.9 & & & $21 X-1,150$ & 197.2 & $21 X-3,90$ & 197.2 & & \\
\hline $\mathrm{w}_{1}^{2}$ & & & $24 X-2,60$ & 223.4 & & & $22 X-3,45$ & 211.05 & $22 \times-6,50$ & 211 & & \\
\hline $\mathrm{w}_{2}$ & & & $24 X-3,100$ & 225.3 & & & $22 X-4,120$ & 213.3 & & & & \\
\hline$x_{1}^{2}$ & & & $25 \mathrm{X}-3,125$ & 234.75 & & & & & $23 X-3,130$ & 217 & $13 X-3,25$ & 118.65 \\
\hline $\mathrm{x}_{2}$ & & & $25 X-5,65$ & 237.15 & & & $23 \mathrm{X}-2,120$ & 218.7 & $23 \mathrm{X}-5,45$ & 219.15 & $13 X-4,110$ & 121 \\
\hline $\mathrm{Y}_{1}$ & & & $26 \times-3,50$ & 243.6 & & & $23 \times-6,55$ & 224.05 & $24 X-3,45$ & 224.45 & $14 X-3,80$ & 128.8 \\
\hline$Y_{2}$ & & & $26 \times-4,90$ & 245.5 & & & $23 \times-7,77$ & 225.77 & $24 X-4,75$ & 226.25 & $14 \times-4,120$ & 130.7 \\
\hline $\mathrm{z}_{1}^{2}$ & & & & & & & & & $27 \mathrm{X}-2,120$ & 249.2 & $15 X-5,90$ & 141.6 \\
\hline $\mathrm{z}_{2}$ & & & & & & & & & $29 X-1,110$ & 267 & $18 X-6,75$ & 171.95 \\
\hline $\mathrm{Z}_{3}$ & & & & & & & & & $31 X-2,120$ & 288 & $19 X-6,120$ & 182.1 \\
\hline $\mathrm{z}_{4}$ & & & & & & & & & $35 X-4,55$ & 329.05 & $25 X-4,80$ & 236.9 \\
\hline $\mathrm{Z}_{5}$ & & & & & & & & & $37 \times-3,40$ & 346.8 & $27 X-4,60$ & 256 \\
\hline $\mathrm{z}_{6}$ & & & & & & & & & $38 \times-4,65$ & 358.25 & $28 X-4,75$ & 265.85 \\
\hline
\end{tabular}

below the contact. The sedimentary sequence below the contact is extremely light color dominant which appears only in the sequence below marker horizon OR-W 1 . The thickness for the missing interval at Site 731 is calculated as $80.80 \mathrm{~m}$ based on occurrence of the markers at Site 721 and as $74.90 \mathrm{~m}$ at Site $\mathbf{7 2 2}$.

The Owen Ridge is isolated high in the northwestern Indian Ocean, and the accumulation of the pelagic sediments may increase gravitational instability which can induce submarine slides. Site 721 sits on the flat ridge top, Site 722 is located on the western margin of the flat top, and Site 731 lies on the upper part of the western slope of the Owen Ridge (Fig. 1). The locations of the drilled sites are consistent with the number of hiatuses; slope and crest margin sites can be assumed as being more unstable for gravity slides. The submarine slide surfaces have been detected on the seismic profiles (Prell, Niitsuma, et al., 1989). The sliding surfaces coincide with the top of the sedimentary sequence with massive light color layers, which contain more than $70 \% \mathrm{CaCO}_{3}$ (Prell, Niitsuma, et al., 1989), and the missing sedimentary interval with the hiatuses is stratified alternation of light and dark layers with thickness thinner than $1 \mathrm{~m}$. The lithological difference of the sedimentary sequence might be related to the mechanical strength for the gravity slide.

The rates of sedimentation are same above the marker horizon OR- $\mathrm{D}_{4}$ in the Owen Ridge Sites, 721, 722, and 731, shown as overlaying lines on the graph (Fig. 3); however, the correlation line between Sites 721 and 731 has a steeper gradient than that between Sites 721 and 722 in the interval from OR-D ${ }_{4}$ to the hiatus below $\mathrm{OR}-\mathrm{H}_{1}$. The difference in the relative rate of sedimentation in Site 731 indicates that the rate of sedimentation was higher in Site 731 than the other part of the Ridge for filling the depression, formed by the gravity slide.

\section{Spatial Changes in the Rate of Sedimentation in the Oman Margin}

Figure 4 shows the comparison of the stratigraphic depth of all correlative marker horizons of Sites $724,727,728$, and 730 against that of Site 723 on the Oman Margin. The gradients of lines represent their relative rates of sedimentation. 
Table 3. Core top depth (mbsf) of the Owen Ridge holes.

\begin{tabular}{|c|c|c|c|c|c|c|}
\hline $\begin{array}{c}\text { Core } \\
\text { no. }\end{array}$ & $\begin{array}{l}\text { Hole } \\
721 \mathrm{~A}\end{array}$ & $\begin{array}{l}\text { Hole } \\
721 \mathrm{~B}\end{array}$ & $\begin{array}{l}\text { Hole } \\
721 \mathrm{C}\end{array}$ & $\begin{array}{l}\text { Hole } \\
722 \mathrm{~A}\end{array}$ & $\begin{array}{l}\text { Hole } \\
722 B\end{array}$ & $\begin{array}{l}\text { Hole } \\
731 \mathrm{~A}\end{array}$ \\
\hline 1 & 2.8 & 0 & 0.1 & 1.8 & 0 & 0 \\
\hline 2 & 12.7 & 9.4 & 4.1 & 11 & 5.8 & 9.8 \\
\hline 3 & 22.9 & 19.6 & 14.2 & 20.8 & 15.4 & 19.3 \\
\hline 4 & 33.6 & 29.5 & 24.1 & 31.1 & 25.5 & 28.8 \\
\hline 5 & 44 & 40.1 & 34.3 & 40.6 & 35.3 & 38.3 \\
\hline 6 & 54.4 & 50.1 & 44.2 & 50.5 & 45 & 47.8 \\
\hline 7 & 63.8 & 59.1 & 54.1 & 60.8 & 55.6 & 57.3 \\
\hline 8 & 73.3 & 68.2 & 64.3 & 70.9 & 65.5 & 67 \\
\hline 9 & 83.9 & 77.9 & 72.1 & 81.1 & 75.5 & 76.7 \\
\hline 10 & & 87.8 & 83.1 & 90.7 & 84.3 & 86.4 \\
\hline 11 & & 97 & 92.8 & 98.6 & 94.1 & 96 \\
\hline 12 & & 104.9 & 99.7 & 109.5 & 103.6 & 105.7 \\
\hline 13 & & 109.1 & 109.1 & 118.5 & 113.3 & 115.4 \\
\hline 14 & & 117 & 114.7 & 129.1 & 123 & 125 \\
\hline 15 & & 126.7 & 121.6 & 137.8 & 132.6 & 134.7 \\
\hline 16 & & 143.8 & & 147.9 & 142.3 & 144.4 \\
\hline 17 & & 153.5 & & 157 & 152.5 & 154 \\
\hline 18 & & 163.2 & & 166.6 & 162.1 & 163.7 \\
\hline 19 & & 172.9 & & 176.3 & 175.9 & 173.4 \\
\hline 20 & & 182.6 & & 186 & 183.7 & 183.1 \\
\hline 21 & & 192.3 & & 195.7 & 193.3 & 192.8 \\
\hline 22 & & 202 & & 207.6 & 203 & 202.5 \\
\hline 23 & & 211.7 & & 216 & 212.7 & 212.2 \\
\hline 24 & & 221.3 & & & 221 & 221.9 \\
\hline 25 & & 230.5 & & & 227.2 & 231.6 \\
\hline 26 & & 240.1 & & & 236.9 & 241.3 \\
\hline 27 & & 249.8 & & & 246.5 & 250.9 \\
\hline 28 & & 259.6 & & & 256.2 & 260.6 \\
\hline 29 & & 269.4 & & & 265.9 & 270.2 \\
\hline 30 & & 279.2 & & & 275.6 & 279.9 \\
\hline 31 & & 289 & & & 285.3 & 289.6 \\
\hline 32 & & 298.8 & & & 295 & 299.2 \\
\hline 33 & & 308.4 & & & 304.7 & 308.9 \\
\hline 34 & & 318 & & & 314.3 & 315.5 \\
\hline 35 & & 327.7 & & & 324 & 322.1 \\
\hline 36 & & 337.4 & & & 333.7 & 331.7 \\
\hline 37 & & 347 & & & 343.4 & 341.3 \\
\hline 38 & & 356.6 & & & 353.1 & 351 \\
\hline
\end{tabular}

The Oman Margin is typical continental borderland and consists of sedimentary basins and their outer sills. The outer sills are being tectonically uplifted by en-echelon anticlines (Fig. 1). The subsiding zones are formed along the inner portion of the uplifted sills, and sedimentary basins are formed, because the subsidence allows continuous accumulation of sediments and the outer sill dams up the transported sediments. The tectonic movement relates to the right lateral movement between the Arabian Peninsula and Indian Ocean along the continental margin. Sites 723 and 727 are located on the depocenter of the sedimentary basin, Sites 724 and 728 are located on the inner slope of the uplifted zones, and Site 730 is located on the uplifting sill.

A sedimentation rate is controlled by the balance of the supply and removal of sediments; this balance is critically controlled by submarine topography. Based on the seismic profiles, the thickness of a layer changes laterally with the topographic relief of the seafloor; the steeper slopes have thinner layers and the gentler slopes have thicker layers. The critical gradient of the slope is $2.2^{\circ}$, above which deposition does not occur in the trench slope area of the Japan Trench (Niitsuma and Akiba, 1985). The framework of the submarine topography along the Oman Margin is constructed by the tectonic movements of subsiding basins and uplifting sill. If subsidence of the tectonic basin would exceed the sediment supply, a topographic depression would be formed on the basin; however, such a depression cannot be found on the Oman Margin (Mountain and Prell, 1989), which means that the sediment supply is enough to fill the subsiding basins and an excess of the sediments is carried away.

Site 723 is located on the axis of the subsiding basin with highest rate of sedimentation, $175 \mathrm{~m} / \mathrm{m}$.y. on the average (Niitsuma et al., this volume). Site 727 is located on the south extension of the subsiding axis with a sedimentation rate of $108 \mathrm{~m} /$ m.y. Sites 724 and 728 are located on the inner slopes of the tectonically uplifting sills and Site 730 sits on the uplifting sill, with sedimentation rates of $90 \mathrm{~m} / \mathrm{m} . y ., 48 \mathrm{~m} / \mathrm{m} . y$., and $44 \mathrm{~m}$ / m.y., respectively. The differences of the sedimentation rates are consistent with the tectonic settings of the sites.

The points which represent depths of the marker horizons of Site 727 are on a nearly straight line (Fig. 4). The straight line

Table 4. Horizon and description of marker horizons for layer-by-layer correlation in the Oman Margin (bioturbation; size in millimeters shown in parenthesis).

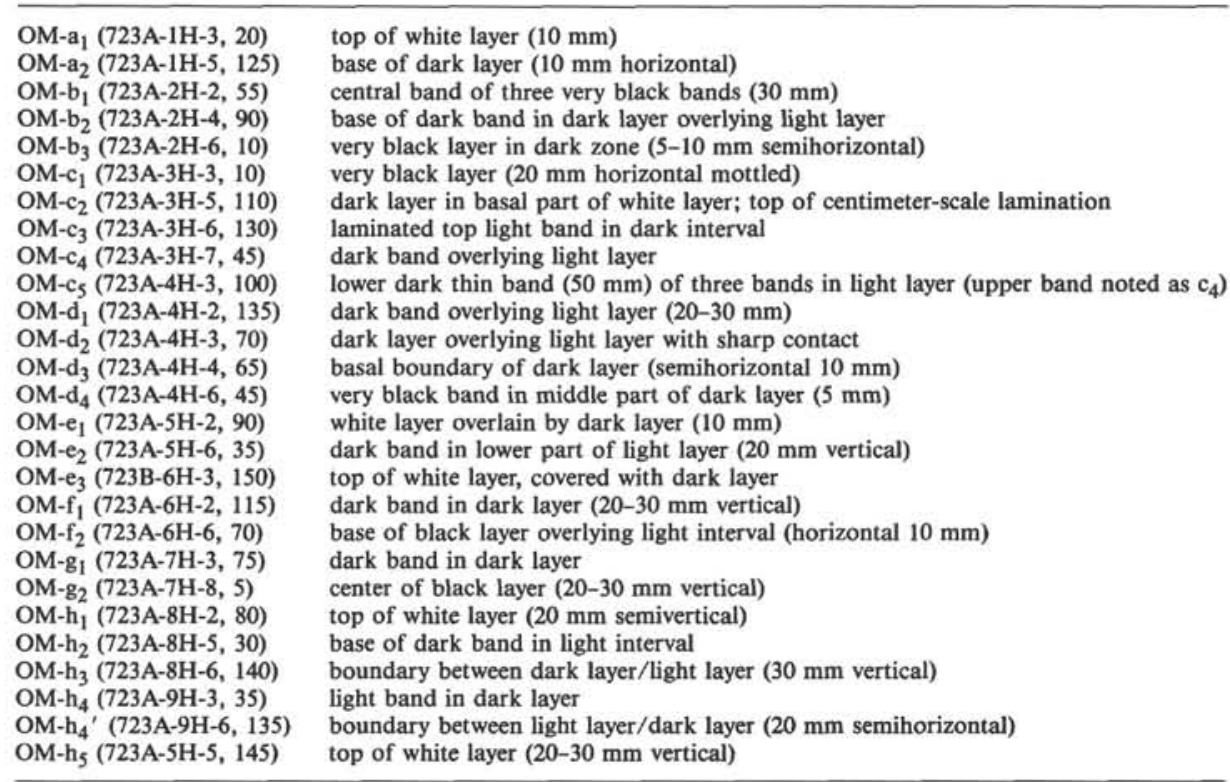


connection between Sites 723 and 727 means that the rate of sedimentation changed on same trend and the ratio of the sedimentation rate was nearly constant. The correlation lines for the other sites are not straight but curve simultaneously. The steeper part of the line represents increase of the sedimentation rate relative to that of Site 723. Based on the oxygen isotope stratigraphy (Fig. 4; Niitsuma et al., this volume), the steeper parts correspond generally to glacial stages, given by even stage numbers and the gentle parts to the interglacial stages (Fig. 4). The contrast in the rate of sedimentation is clearly shown in the interval younger than Stage 8, especially Stages 5, 6, and 7. Because Site 723 has more than two times higher sedimentation rate in the glacial stages based on the oxygen isotope stratigraphy (Fig. 4), the increases of sedimentation rate on the Oman Margin should be more than four times larger in the glacial stages.

Figure 4 shows that Sites 723 and 727 on the subsiding axis are affected by the climatic cycles less than Sites 724, 728, and 730 on the slope and top of uplifting sill. The sedimentation rate in the subsiding basin is controlled by the amount of the relative subsidence and the sediment supply; on the other hand, the rate on the sill is controlled by the sediment supply and the gradient of the seafloor, as mentioned above. It means that the rates on the sill are more sensitive for the sediment supply than that in the subsiding basin and their fluctuations are caused by the changes in the sediment supply. Because the time constant of the tectonic movements can be thought of as much longer than that of glacial-interglacial climatic cycles, the changes in the rate of sedimentation might be affected by the amount of the sediment supply on the Oman Margin, modulated by the climatic cycles; i.e., the sediment supply increased in glacial stages and decreased in the interglacial stages. The increases are accompanied with high magnetic susceptibility of the sediments (Fig. 4; Prell, Niitsuma et al., 1989).

The marker horizons are found more in the glacial stages than in interglacial stages, especially two marker horizons in Stages 5 and eight marker horizons in Stage 6, because of the variable lithofacies in the sedimentary sequence. The difference means that glacial stage had not only greater sediment supply but also greater variability.

\section{COMPARISON OF COLOR DENSITY PATTERN}

The marker horizons allow us to compare the trend of the color density pattern within the drilling areas of the Oman Margin and Owen Ridge, and the trend can be compared with magnetic susceptibility curve and other characters. Furthermore, by using the oxygen isotope stratigraphic correlation between the Owen Ridge, Oman Margin, and Indus Fan, the origin of the trend and marker layers can be estimated. Figure 5 shows the color density patterns of sediments drilled during Leg 117 with the positions of the marker horizons and oxygen isotope stratigraphic stages younger than Stage 15 . The vertical scale for each site is extended or reduced for the comparison; the degree of the extension or reduction is represented by the length of the bar on the top left of each graph, which corresponds to $5 \mathrm{~m}$ of thickness. The longer bar represents lower rate of sedimentation. The vertical line in each graph shows the value of 150 in the 256 gradations of color density, measured with video-densitometer; real black and real white on black and white photograph are counted as 30 and 220 of the gradation, respectively.

The darkness of the color density is completely different in the Oman Margin, the Owen Ridge, and the Indus Fan. Oman Margin sediments have darker color than the Owen Ridge and Indus Fan. The increase in darkness on the graph of the Indus Fan in the lower part is affected by the turbiditic terrigenous materials (Prell, Niitsuma, et al., 1989). Generally the color density is controlled by the contents of $\mathrm{CaCO}_{3}$ and organic carbon. Higher $\mathrm{CaCO}_{3}$ and lower organic carbon contents give lighter color density, higher value of the gradation. The $\mathrm{CaCO}_{3}$ contents of the sediments range from $43 \%$ to $73 \%$ for the Oman Margin, from $55 \%$ to $78 \%$ for the Owen Ridge, and from $35 \%$ to $68 \%$ for the Indus Fan; the organic carbon contents range from $0.3 \%$ to $6.8 \%$ for the Oman Margin, from $0.1 \%$ to $1.9 \%$ for the Owen Ridge, and from $0.3 \%$ to $2.3 \%$ for the Indus Fan (Prell, Niitsuma, et al., 1989). These contents of $\mathrm{CaCO}_{3}$ and organic carbon are consistent with the distinct difference in the color densities; however, the ranges of the contents overlap, and a simple numerical relationship between the color density and these contents cannot be applied for these sites.

The content of organic carbon in the Oman Margin sediments is directly related to high productivity in the upwelling area induced by the southwest monsoon. Because the drilled sites in the Oman Margin are located in the oxygen minimum zone, the organic carbon can be preserved in sediments. The sites in the Owen Ridge are located below the oxygen minimum zone, and most of the organic carbon has been oxidized before reaching the sediments. So, the organic carbon content does not control the color density in these deeper sites. Color densities of these sites may result from the difference in the main control factor for the color density fluctuations; organic carbon content in the Oman Margin sediments (Niitsuma et al., this volume) and the content of terrigenous matter in the Owen Ridge sediments (Murray and Prell, this volume). These factors are directly controlled by the flux of terrigenous matter, which was changed during the glacial-interglacial cycle. The flux with intermediate color density of $\mathrm{CaCO}_{3}$ and organic carbon increased greatly during glacial stages, as mentioned above. The difference in the fluctuations of the color densities appears clearly in Stage 6 with contrast to Stage 5. In Site 723 of the Oman Margin, darker colors dominate in the interglacial Stage 5 rather than in the glacial Stage 6; however, the inverse relation occurs in the Owen Ridge sites. The other sites of the Oman Margin have intermediate character in the fluctuations of color densities (Fig. 5).

The durations of Stage 5 and Stage 6 are almost same; 57 k.y. and 58 k.y., respectively, based on SPECMAP time table (Imbrie et al., 1984). However, the intervals of Stage 6 are significantly thicker than that of Stage 5 in all sites and have remarkable changes laterally in the Oman Margin (Fig. 5). The differences of the thickness mean that the rate of sedimentation was significantly higher in the glacial Stage 6 than interglacial Stage 5. The lateral changes are provided by the light layer intercalated between marker horizons $\mathrm{OM}-\mathrm{c}_{5}$ and $\mathrm{OM}-\mathrm{d}_{1}$ in the middle part of Stage 6. The bioturbations in the lighter layers do not occur or they develop less than in the darker layers. The difference indicates that the lighter layer was supplied more rapidly than the darker layer and the thickness could be more sensitive to the bottom surface condition of the seafloor.

The contrast of the color density in the glacial-interglacial cycles becomes less clear before oxygen isotope Stage 8 . This trend of the feature is similar to that of sedimentation rate and magnetic susceptibility (Fig. 4). These indicate that the present distinct climatic cycle relating to the southwest monsoon has been developed since Stage 8 .

\section{SEDIMENT SUPPLY DURING GLACIAL AND INTERGLACIAL CLIMATIC CYCLE}

The layer-by-layer correlation demonstrated a distinct contrast in the amount of sediment supply during glacial and interglacial stages; larger supply occurred in the glacial stages, as mentioned above. The increases in the sediment supply were accompanied by greater magnetic susceptibility. The fluctuation of the susceptibility is much larger on the Owen Ridge than the Oman Margin (Prell, Niitsuma, et al., 1989). The amplitude of 
Table 5. Depths of marker horizons in the Oman Margin holes (see text for explanation of gaseous expansion).

\begin{tabular}{|c|c|c|c|c|c|c|c|c|c|c|c|}
\hline $\begin{array}{l}\text { Marker } \\
\text { horizon }\end{array}$ & $\begin{array}{l}\text { Hole 723B } \\
\text { Core, section, } \\
\text { interval }(\mathrm{cm})\end{array}$ & Gas & $\begin{array}{l}\text { Depth } \\
\text { (mbsf) }\end{array}$ & $\begin{array}{l}\text { Hole } 723 \mathrm{~A} \\
\text { Core, section, } \\
\text { interval }(\mathrm{cm})\end{array}$ & Gas & $\begin{array}{l}\text { Depth } \\
\text { (mbsf) }\end{array}$ & $\begin{array}{l}\text { Hole } 724 \mathrm{~A} \\
\text { Core, section, } \\
\text { interval }(\mathrm{cm})\end{array}$ & $\begin{array}{l}\text { Depth } \\
\text { (mbsf) }\end{array}$ & $\begin{array}{l}\text { Hole 724B } \\
\text { Core, section, } \\
\text { interval }(\mathrm{cm})\end{array}$ & $\begin{array}{l}\text { Depth } \\
\text { (mbsf) }\end{array}$ & $\begin{array}{c}\text { Hole } 724 \mathrm{C} \\
\text { Core, section, } \\
\text { interval }(\mathrm{cm})\end{array}$ \\
\hline$O M-a_{1}$ & $1 \mathrm{H}-3,20$ & & 3.2 & $1 \mathrm{H}-3,35$ & & 3.35 & $1 \mathrm{H}-1,95$ & 0.95 & $1 \mathrm{H}-1,95$ & 0.95 & $1 \mathrm{H}-1,110$ \\
\hline$a_{2}$ & $1 \mathrm{H}-5,125$ & & 7.25 & $2 \mathrm{H}-3,20$ & & 7.2 & $1 \mathrm{H}-2,115$ & 2.65 & $1 \mathrm{H}-2,105$ & 2.55 & \\
\hline$b_{1}$ & $2 \mathrm{H}-2,55$ & & 9.25 & $2 \mathrm{H}-4,65$ & & 9.15 & $1 \mathrm{H}-3,100$ & 4 & $1 \mathrm{H}-3,100$ & 4 & $2 \mathrm{H}-1,115$ \\
\hline$b_{2}$ & $2 \mathrm{H}-4,90$ & & 12.6 & $2 \mathrm{H}-6,110$ & & 12.6 & $1 \mathrm{H}-4,95$ & 5.45 & $1 \mathrm{H}-4,105$ & 5.55 & $2 \mathrm{H}-2,115$ \\
\hline$b_{3}$ & $2 \mathrm{H}-6,10$ & & 14.8 & $3 \mathrm{H}-1,95$ & & 14.75 & & & & & $2 \mathrm{H}-4,60$ \\
\hline$c_{1}$ & $3 \mathrm{H}-3,10$ & & 20.9 & $3 \mathrm{H}-5,110$ & & 20.9 & $2 \mathrm{H}-2,75$ & 9.45 & $2 \mathrm{H}-2,85$ & 9.45 & \\
\hline$c_{2}$ & $3 \mathrm{H}-5,110$ & & 24.9 & $4 \mathrm{H}-1,85$ & & 24.95 & $2 \mathrm{H}-3,130$ & 11.5 & $2 \mathrm{H}-3,130$ & 11.4 & $2 \mathrm{H}-6,115$ \\
\hline$c_{3}$ & $3 \mathrm{H}-6,130$ & & 26.6 & $4 \mathrm{H}-2,100$ & & 26.6 & $2 \mathrm{H}-4,70$ & 12.4 & $2 \mathrm{H}-4,75$ & 12.35 & $2 \mathrm{H}-7,50$ \\
\hline$c_{4}$ & $3 \mathrm{H}-7,45$ & & 27.25 & $4 \mathrm{H}-3,15$ & & 27.25 & $2 \mathrm{H}-4,120$ & 12.9 & $2 \mathrm{H}-4,135$ & 12.95 & \\
\hline$c_{5}$ & & & & $1 \mathrm{H}-3,100$ & & 28.1 & $2 \mathrm{H}-5,35$ & 13.55 & $2 \mathrm{H}-5,50$ & 13.6 & $3 \mathrm{H}-1,50$ \\
\hline $\mathrm{d}_{1}$ & $4 \mathrm{H}-2,135$ & & 30.75 & $4 \mathrm{H}-5,70$ & & 30.8 & $3 \mathrm{H}-1,40$ & 17.2 & $3 \mathrm{H}-1,45$ & 17.15 & $3 \mathrm{H}-3,115$ \\
\hline $\mathrm{d}_{2}$ & $4 \mathrm{H}-3,70$ & & 31.6 & $4 \mathrm{H}-6,10$ & & 31.7 & $3 \mathrm{H}-1,150$ & 18.3 & $3 \mathrm{H}-2,5$ & 18.25 & $3 \mathrm{H}-4,65$ \\
\hline $\mathrm{d}_{3}$ & $4 \mathrm{H}-4,65$ & & 33.05 & $4 \mathrm{H}-7,5$ & & 33.15 & $3 \mathrm{H}-2,90$ & 19.2 & $3 \mathrm{H}-2,105$ & 19.25 & $3 \mathrm{H}-5,20$ \\
\hline$d_{4}$ & $4 \mathrm{H}-6,45$ & & 35.85 & $5 \mathrm{H}-2,50$ & 2 & 35.88 & $3 \mathrm{H}-3,85$ & 20.65 & $3 \mathrm{H}-3,105$ & 20.75 & $3 \mathrm{H}-6,20$ \\
\hline$e_{1}$ & $5 \mathrm{H}-2,90$ & 12 & 39.88 & $5 \mathrm{H}-5,20$ & 3 & 10.07 & $3 \mathrm{H}-4,15$ & 21.45 & $3 \mathrm{H}-4,35$ & 21.55 & $3 \mathrm{H}-6,105$ \\
\hline$e_{2}$ & $5 \mathrm{H}-6,35$ & 23 & 45.22 & $6 \mathrm{H}-1,90$ & & 45.2 & $3 \mathrm{H}-5,90$ & 23.7 & $3 \mathrm{H}-5,115$ & 23.85 & $4 \mathrm{H}-1,80$ \\
\hline$e_{3}$ & & & & $6 \mathrm{H}-3,150$ & 3 & 48.77 & $3 \mathrm{H}-6,95$ & 25.25 & $3 \mathrm{H}-6,135$ & 25.55 & $4 \mathrm{H}-2,100$ \\
\hline$f_{1}$ & $6 \mathrm{H}-2,115$ & 48 & 51.47 & $6 \mathrm{H}-5,120$ & 10 & 51.4 & & & & & $4 \mathrm{H}-3,115$ \\
\hline$f_{2}$ & $6 \mathrm{H}-6,70$ & 61 & 56.89 & $7 \mathrm{H}-2,15$ & 12 & 56.63 & $4 \mathrm{H}-2,70$ & 29.1 & $4 \mathrm{H}-2,45$ & 29.15 & $4 \mathrm{H}-5,10$ \\
\hline$g_{1}$ & $7 \mathrm{H}-3,75$ & 145 & 62.8 & $7 \mathrm{H}-6,30$ & 18 & 62.72 & $4 \mathrm{H}-4,120$ & 32.6 & $4 \mathrm{H}-4,95$ & 32.65 & $4 \mathrm{H}-7,85$ \\
\hline $\mathrm{g}_{2}$ & $7 \mathrm{H}-8,5$ & 229 & 68.76 & $8 \mathrm{H}-2,60$ & 32 & 68.78 & $4 \mathrm{H}-6,25$ & 34.65 & $4 \mathrm{H}-6,5$ & 34.75 & $5 \mathrm{H}-1,150$ \\
\hline $\mathrm{h}_{1}$ & $8 \mathrm{H}-2,80$ & 3 & 73.17 & $8 \mathrm{H}-5,90$ & 83 & 73.07 & $4 \mathrm{H}-7,40$ & 36.3 & $5 \mathrm{H}-1,70$ & 36.3 & $5 \mathrm{H}-3,10$ \\
\hline $\mathrm{h}_{2}$ & $8 \mathrm{H}-5,30$ & 26 & 76.94 & $9 \mathrm{H}-1,20$ & & 76.3 & $5 \mathrm{H}-1,80$ & 37.3 & $5 \mathrm{H}-2,20$ & 37.3 & $5 \mathrm{H}-3,100$ \\
\hline $\mathrm{h}_{3}$ & $8 \mathrm{H}-6,140$ & 26 & 79.54 & $9 \mathrm{H}-3,135$ & 93 & 79.52 & $5 \mathrm{H}-2,60$ & 38.6 & $5 \mathrm{H}-2,150$ & 38.6 & $5 \mathrm{H}-4,85$ \\
\hline $\mathrm{h}_{4}$ & $9 \mathrm{H}-3,35$ & 43 & 85.12 & $9 \mathrm{H}-7,95$ & 97 & 85.08 & $6 \mathrm{H}-4,145$ & 42.45 & $5 \mathrm{H}-5,90$ & 42.5 & $5 \mathrm{H}-7,20$ \\
\hline $\mathrm{h}_{4}$ & $9 \mathrm{H}-6,135$ & 78 & 90.27 & $10 X-4,70$ & 136 & 90.34 & & & & & \\
\hline $\mathrm{h}_{5}$ & & & & & & & SH-5, 145 & 48.95 & $5 \mathrm{H}-6,90$ & 44 & $6 X-1,60$ \\
\hline
\end{tabular}

the fluctuations of the magnetic susceptibility increase at oxygen isotope Stages 8 . The changes in the magnetic susceptibility indicate that the distinct contrast of the amount of sediment supply started from Stage 8 (about $250 \mathrm{ka}$ ).

Oxygen and carbon isotopic data demonstrate that stronger upwelling developed on the Oman Margin in the interglacial stages than in glacial stages (Niitsuma et al., this volume). This relation means that glacial stages had weaker upwelling yet a larger influx of sediments. If the sediments were transported by the southwest monsoon which induces upwelling in the Oman Margin, there should be an inverse relation between them. For this reason, the supply of a large amount of glacial-aged sediments on the Oman Margin can be assumed as fluvial origin, not eolian origin. The fluvial origin sediments can be controlled by the drainage systems on land and can have variability; this is consistent with the various lithofacies in the glacial stages, as mentioned above.

Meteorological studies show that the southwest monsoon is induced by the strong ascending current on the Himalayas-Tibet high-mountainous area in summer, and the counter-current of the southwest monsoon develops the Tropical Easterly Jet from southeast Asia to north Africa (Hahn and Manabe, 1975; Barnett et al., 1989). The Easterly Jet is directly related to the high precipitation in southeast Asia and arid climate in North Africa and the Arabian Peninsula. If the relation between the strong monsoon and arid climate of the Arabian Peninsula can also be assumed for the past, the glacial stage with weak upwelling had more humid climate in the Arabian Peninsula. The relation is much more in agreement with the fluvial origin of the sediments in the glacial stages.

Although Kutzbach (1986) mentioned that lake-levels in Africa were low in the last glacial with a weaker monsoon, based on Butzer et al. (1972) and Street and Grove (1979), humid conditions in the last glacial stage have been reported from the lake sediments of the Dead Sea and Zeribar in the Middle East and Lake Mundafan in the Arabian Peninsula (Street and Grove, 1979). Lake deposits of Lake Mundafan in the central part of the Arabian Peninsula were classified into Series A, B, and C based on their radiocarbon dates, and their dates are 36-17 ka, 9-6 ka, and 15-11 ka, respectively. Generally, beds of Series A occupy high parts of the basin and beds of Series B and Series C occupy low parts (McClure, 1976). The lake deposits indicate much more humid conditions in the last glacial than present and support the fluvial origin of the sediments supply in glacial stages.

\section{SUMMARY AND CONCLUSION}

This paper has given the method of layer-by-layer correlation and shore-based studies, nomenclature system to be adopted in this work, description of the marker horizons in sediment cores taken from Owen Ridge and Oman Margin, and discussion on the paleoclimatological and sedimentological phenomena in the northwestern Indian Ocean based on the layer-by-layer correlation.

Interhole correlations were carried out by comparing core photographs based on their color, thickness, and other characteristics. One hundred thirty-one marker horizons relating to the distinct and traceable layers were described with the notation OR-A $A_{0}, A_{1}, A_{2}, \ldots, Z_{5}, Z_{6}$ for the Owen Ridge sites and OM- $\mathrm{a}_{1}, \mathrm{a}_{2}, \ldots, \mathrm{h}_{4}, \mathrm{~h}_{5}$ for the Oman Margin sites. The correlations utilized the calculations of true depth, corrected for coring disturbance and gas expansion. Intersite correlation of marker horizons has been improved based on color density data, measured with video densitometer, and oxygen isotope stratigraphic data.

Distinct hiatuses were detected by intersite correlation of the marker horizons on the Owen Ridge. The hiatuses are related to submarine slides induced by increasing gravitational instability resulting from the accumulation of the pelagic sediments at the rate of $35-48 \mathrm{~m} / \mathrm{m}$.y. on the top of isolated high, the Owen Ridge.

In the Oman Margin, Site 723 is located at the axis of the subsiding basin with highest rate of sedimentation, $175 \mathrm{~m} / \mathrm{m}$.y. on average. Site 727 is located on the south extension of the subsiding axis with a sedimentation rate of $108 \mathrm{~m} / \mathrm{m}$.y. Sites 724 
Table 5 (continued).

\begin{tabular}{|c|c|c|c|c|c|c|c|c|c|c|}
\hline $\begin{array}{l}\text { Depth } \\
\text { (mbsf) }\end{array}$ & $\begin{array}{l}\text { Hole } 727 \mathrm{~A} \\
\text { Core, section, } \\
\text { interval }(\mathrm{cm})\end{array}$ & $\begin{array}{l}\text { Depth } \\
\text { (mbsf) }\end{array}$ & $\begin{array}{l}\text { Hole } 727 \mathrm{~B} \\
\text { Core, section, } \\
\text { interval }(\mathrm{cm})\end{array}$ & $\begin{array}{l}\text { Depth } \\
\text { (mbsf) }\end{array}$ & $\begin{array}{c}\text { Hole } 728 \mathrm{~A} \\
\text { Core, section, } \\
\text { interval }(\mathrm{cm})\end{array}$ & $\begin{array}{l}\text { Depth } \\
\text { (mbsf) }\end{array}$ & $\begin{array}{c}\text { Hole } 728 \mathrm{~B} \\
\text { Core, section, } \\
\text { interval }(\mathrm{cm})\end{array}$ & $\begin{array}{l}\text { Depth } \\
\text { (mbsf) }\end{array}$ & $\begin{array}{l}\text { Hole } 730 \mathrm{~A} \\
\text { Core, section, } \\
\text { interval }(\mathrm{cm})\end{array}$ & $\begin{array}{l}\text { Depth } \\
\text { (mbsf) }\end{array}$ \\
\hline \multirow[t]{2}{*}{1.1} & & & $1 \mathrm{H}-2,15$ & 1.65 & & & $1 \mathrm{H}-1,65$ & 0.65 & & \\
\hline & $1 \mathrm{H}-1,115$ & 4.25 & $1 \mathrm{H}-3,125$ & 4.25 & $1 \mathrm{H}-2,35$ & 2.25 & $2 \mathrm{H}-1,100$ & 2.2 & $1 \mathrm{H}-1,120$ & 1.2 \\
\hline 3.95 & $1 \mathrm{H}-3,50$ & 6.6 & $1 \mathrm{H}-5,60$ & 6.6 & $1 \mathrm{H}-2,90$ & 2.8 & $2 \mathrm{H}-2,5$ & 2.75 & $1 \mathrm{H}-2,40$ & 1.9 \\
\hline 5.45 & $1 \mathrm{H}-4,90$ & 8.5 & $2 \mathrm{H}-1,120$ & 8.8 & $1 \mathrm{H}-3,20$ & 3.6 & $2 \mathrm{H}-2,80$ & 3.5 & $1 \mathrm{H}-3,20$ & 3.2 \\
\hline \multirow[t]{2}{*}{7.90} & $1 \mathrm{H}-5,80$ & 9.9 & $2 \mathrm{H}-2,110$ & 10.2 & $1 \mathrm{H}-3,60$ & 4 & $2 \mathrm{H}-2,120$ & 3.9 & $1 \mathrm{H}-3,50$ & 3.5 \\
\hline & $2 \mathrm{H}-1,50$ & 14 & $2 \mathrm{H}-5,40$ & 14 & $1 \mathrm{H}-4,40$ & 5.3 & $2 \mathrm{H}-3,110$ & 5.3 & $1 \mathrm{H}-3,115$ & 4.15 \\
\hline 11.45 & $2 \mathrm{H}-2,125$ & 16.25 & $2 \mathrm{H}-6,115$ & 16.25 & $1 \mathrm{H}-5,10$ & 6.5 & $2 \mathrm{H}-4,85$ & 6.55 & $1 \mathrm{H}-4,35$ & 4.85 \\
\hline \multirow[t]{2}{*}{12.3} & $2 \mathrm{H}-3,95$ & 17.45 & & & $1 \mathrm{H}-5,45$ & 6.85 & $2 \mathrm{H}-4,110$ & 6.8 & $1 \mathrm{H}-4,50$ & 5 \\
\hline & $2 \mathrm{H}-4,0$ & 18 & & & $1 \mathrm{H}-5,75$ & 7.15 & $2 \mathrm{H}-4,135$ & 7.05 & $1 \mathrm{H}-4,75$ & 5.25 \\
\hline 13.5 & $2 \mathrm{H}-4,55$ & 18.55 & & & 1H-5, 105 & 7.45 & $2 \mathrm{H}-5,25$ & 7.45 & $1 \mathrm{H}-4,120$ & 5.7 \\
\hline 17.15 & $2 \mathrm{H}-5,100$ & 20.5 & $3 \mathrm{H}-2,35$ & 20.45 & $1 \mathrm{H}-6,70$ & 8.6 & $2 \mathrm{H}-5,130$ & 8.5 & $1 \mathrm{H}-6,5$ & 7.55 \\
\hline 18.15 & $2 \mathrm{H}-6,15$ & 21.15 & $3 \mathrm{H}-2,100$ & 21.1 & $1 \mathrm{H}-6,115$ & 9.05 & $2 \mathrm{H}-6,30$ & 9 & $1 \mathrm{H}-6,60$ & 8.1 \\
\hline 19.2 & $2 \mathrm{H}-6,110$ & 22.1 & $3 \mathrm{H}-3,55$ & 22.15 & $1 \mathrm{H}-7,15$ & 9.55 & $2 \mathrm{H}-6,95$ & 9.65 & $2 \mathrm{H}-1,35$ & 8.65 \\
\hline 20.7 & $3 \mathrm{H}-1,90$ & 23.9 & $3 \mathrm{H}-4,75$ & 23.85 & & & $2 \mathrm{H}-7,10$ & 10.3 & $2 \mathrm{H}-1,105$ & 9.35 \\
\hline 21.55 & $3 \mathrm{H}-3,5$ & 26.05 & $3 \mathrm{H}-6,5$ & 26.15 & $2 \mathrm{H}-1,110$ & 11.2 & $3 \mathrm{H}-1,40$ & 11.1 & $2 \mathrm{H}-2,5$ & 9.85 \\
\hline 23.8 & $3 \mathrm{H}-5,0$ & 29 & & & $2 \mathrm{H}-2,95$ & 12.55 & $3 \mathrm{H}-2,35$ & 12.55 & $2 \mathrm{H}-3,55$ & 11.85 \\
\hline 25.5 & $3 \mathrm{H}-5,130$ & 30.3 & & & $2 \mathrm{H}-3,60$ & 13.7 & $3 \mathrm{H}-2,145$ & 13.65 & $2 \mathrm{H}-4,40$ & 13.2 \\
\hline 27.15 & $3 \mathrm{H}-7,25$ & 32.25 & & & $2 \mathrm{H}-3,120$ & 14.3 & $3 \mathrm{H}-3,65$ & 14.35 & $2 \mathrm{H}-4,100$ & 13.8 \\
\hline 29.1 & $4 \mathrm{H}-2,95$ & 34.95 & & & $2 \mathrm{H}-4,55$ & 15.15 & $3 \mathrm{H}-4,5$ & 15.25 & $2 \mathrm{H}-5,10$ & 14.4 \\
\hline 32.85 & $4 \mathrm{H}-4,100$ & 38 & & & $2 \mathrm{H}-5,35$ & 16.45 & $3 \mathrm{H}-4,130$ & 16.5 & & \\
\hline 34.7 & $4 \mathrm{H}-7,40$ & 41.9 & & & $2 \mathrm{H}-5,105$ & 17.15 & $3 \mathrm{H}-5,60$ & 17.3 & & \\
\hline 36.3 & $5 \mathrm{H}-4,40$ & 46.8 & & & $2 \mathrm{H}-6,70$ & 18.3 & $3 \mathrm{H}-6,35$ & 18.55 & & \\
\hline 37.2 & $5 \mathrm{H}-6,20$ & 49.6 & & & $2 \mathrm{H}-7,35$ & 19.45 & $3 \mathrm{H}-6,140$ & 19.6 & & \\
\hline 38.55 & SH-6, 135 & 50.75 & & & $3 \mathrm{H}-1,90$ & 20.7 & & & & \\
\hline 42.4 & $6 \mathrm{H}-3,95$ & 55.35 & & & $3 \mathrm{H}-2,65$ & 21.95 & $4 \mathrm{H}-1,120$ & 22 & & \\
\hline 48.9 & $6 \mathrm{H}-4,95$ & 54.85 & & & $3 \mathrm{H}-2,150$ & 22.8 & $4 \mathrm{H}-2,40$ & 22.7 & & \\
\hline
\end{tabular}

Table 6. Core top depth (mbsf) of the Oman Margin holes.

\begin{tabular}{ccccccccccc}
\hline $\begin{array}{c}\text { Core } \\
\text { no. }\end{array}$ & $\begin{array}{c}\text { Hole } \\
723 \mathrm{~A}\end{array}$ & $\begin{array}{c}\text { Hole } \\
723 \mathrm{~B}\end{array}$ & $\begin{array}{c}\text { Hole } \\
724 \mathrm{~A}\end{array}$ & $\begin{array}{c}\text { Hole } \\
724 \mathrm{~B}\end{array}$ & $\begin{array}{c}\text { Hole } \\
724 \mathrm{C}\end{array}$ & $\begin{array}{c}\text { Hole } \\
727 \mathrm{~A}\end{array}$ & $\begin{array}{c}\text { Hole } \\
727 \mathrm{~B}\end{array}$ & $\begin{array}{c}\text { Hole } \\
728 \mathrm{~A}\end{array}$ & $\begin{array}{c}\text { Hole } \\
728 \mathrm{~B}\end{array}$ & $\begin{array}{r}\text { Hole } \\
730 \mathrm{~A}\end{array}$ \\
\hline 1 & 0 & 0 & 0 & 0 & 0 & 3.1 & 0 & 0.4 & 0 & \\
2 & 7.2 & 4 & 7.2 & 7.1 & 2.8 & 13.5 & 7.6 & 10.1 & 1.2 & 8 \\
3 & 17.8 & 13.8 & 16.8 & 16.7 & 13 & 23 & 18.6 & 19.8 & 10.7 & \\
4 & 27.9 & 24.1 & 26.9 & 27.2 & 23 & 32.5 & & & 20.8 & \\
5 & 37.6 & 33.9 & 36.5 & 35.6 & 33.2 & 41.9 & & & & \\
6 & 49.3 & 44.3 & & & 43.3 & 51.4 & & & & \\
7 & 60.5 & 55.1 & & & & & & & & \\
8 & 70.9 & 67 & & & & & & & & \\
9 & 82.2 & 76.1 & & & & & & & & \\
10 & & 86.5 & & & & & & & & \\
11 & & 93.9 & & & & & & & & \\
12 & & 103.4 & & & & & & & & \\
13 & & 113 & & & & & & & & \\
14 & & 122.6 & & & & & & & & \\
15 & & 132.3 & & & & & & & & \\
16 & & 141.9 & & & & & & & & \\
\hline
\end{tabular}

and 728 are located on the inner slopes of tectonically uplifted sills, and Site 730 sits on the uplifting sill, with sedimentation rates of $90 \mathrm{~m} / \mathrm{m} . y ., 48 \mathrm{~m} / \mathrm{m}$.y., and $44 \mathrm{~m} / \mathrm{m}$.y., respectively.

Sites 723 and 727 have rates of sedimentation with the same trend and a constant ratio. The other sites in the Oman Margin have rates with simultaneous cyclic differences from Sites 723 and 727 , a higher rate in the glacial stages and lower rate in the interglacial stages. The difference was derived by the cyclic changes in the amount of sediment flux, because the sedimentation rate in the center of the subsiding basin is controlled mainly by the amount of the sediment supply and the relative subsidence with longer time constant; however, the sedimentation rate on the sill is controlled mainly by the amount of the sediment supply and the gradient of the seafloor rather than general tectonic movement.

Because Site 723 in the glacial stage had more than twice the sedimentation rate of the interglacial stages based on the oxygen isotope stratigraphy, the increases of sediment supply should have been more than four times larger in the glacial stages on the sill of the Oman Margin. This fact might be explained by the large amount of sediment supply during glacial stages.

The color density patterns with glacial-interglacial cycles are controlled by the balance of organic carbon content, increasing in the interglacial stages with strong upwelling induced by the southwest monsoon, and flux of terrigenous matter, increasing in the glacial stages.

The present distinct climatic cycle related to the development of the southwest monsoon has been developed since Stage 8 , $250 \mathrm{ka}$.

The glacial aged sediments can be assumed as having fluvial origin from the humid Arabian Peninsula, relating to the weakened Tropical Easterly Jet, which is induced by the counter-current of the southwest monsoon and which maintains the present arid climate in North Africa and the Arabian Peninsula. 


\section{1 depth (mbsf)}

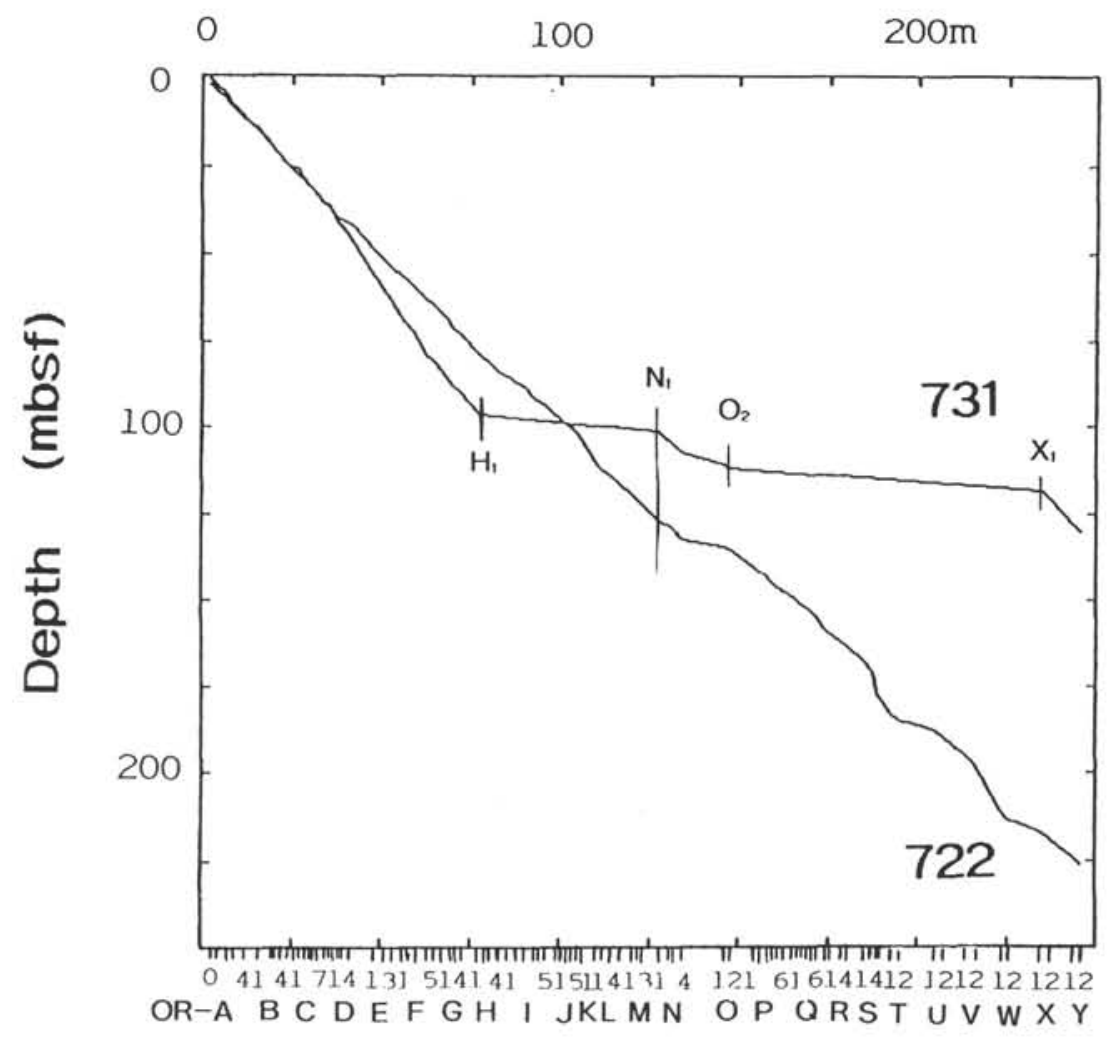

Figure 3. Depth correlation of the marker horizons in the Owen Ridge. Horizontal axis is depth of the marker horizons in Site 721, vertical axis is depth in Sites 722 and 731.

\section{ACKNOWLEDGMENTS}

I thank Warren Prell of Brown University; Philip Meyers of the University of Michigan for critical review, comment, and discussions; Dave Murray of Brown University; Dick Kroon of Vrije University; Tom Pedersen of the University of British Columbia for discussion, comments, and information on oxygen isotope stratigraphic work; John Imbrie and Clemens of Brown University; Susumu Honjo of Wood Hole Oceanographic Institution; Akira Hayashida of Doshisha University; Toshiaki Takayama of Kanazawa University; Hideki Wada of Shizuoka University; Asahiko Taira of University of Tokyo; Tetsuzo Yasunari of Tsukuba University for valuable comments and discussions; and Norman Stewart of ODP for editorial help and improvement of English.

\section{REFERENCES}

Barnett, T. P., Dümenil, L., Schlese, U., Roeckner, E., and Latif, M., 1989. The effect of Eurasian snow cover on regional and global climate variations. J. Atmos. Sci., 46:661-665.
Butzer, K. W., Isaac, G. L., Richardson, J. A., and Washbourn-Kamau, C., 1972. Radiocarbon dating of East African lake levels. Science, 175:1069-1076.

Hahn, D. and Manabe, S., 1975. The role of mountains in the South Asian monsoon circulation. J. Atmos. Sci., 32:1515-1541.

Kutzbach, J. E., 1986. The changing pulse of the Monsoon. In Fein, J. S., and Stephens, P. L. (Eds.), Monsoons: New York (Wiley), 247268.

McClure, H. A., 1976. Radiocarbon chronology of late Quaternary lakes in the Arabian Desert. Nature, 263:756.

Niitsuma, H., and Akiba, F., 1985. Magnetostratigraphy and diatom biostratigraphy of Site 584, Deep Sea Drilling Project Leg 87, and implications for the tectonic evolution of Japanese Island Arcs. In Kagami, H., Karig, D. E., Coulbourn, W. T., et al., Init. Repts. DSDP, 87: Washington (U.S. Govt. Printing Office), 555-571.

Prell, W. L., Niitsuma, N., et al., 1989. Proc. ODP, Init. Repts., 117: College Station, TX (Ocean Drilling Program).

Street, F. A., and Grove, A. T., 1979. Global maps of lake-level fluctuations since 30,000 yr B.P. Quat. Res., 12:83-118.

Date of initial receipt: 3 October 1989

Date of acceptance: 8 May 1990

Ms 117B-139 

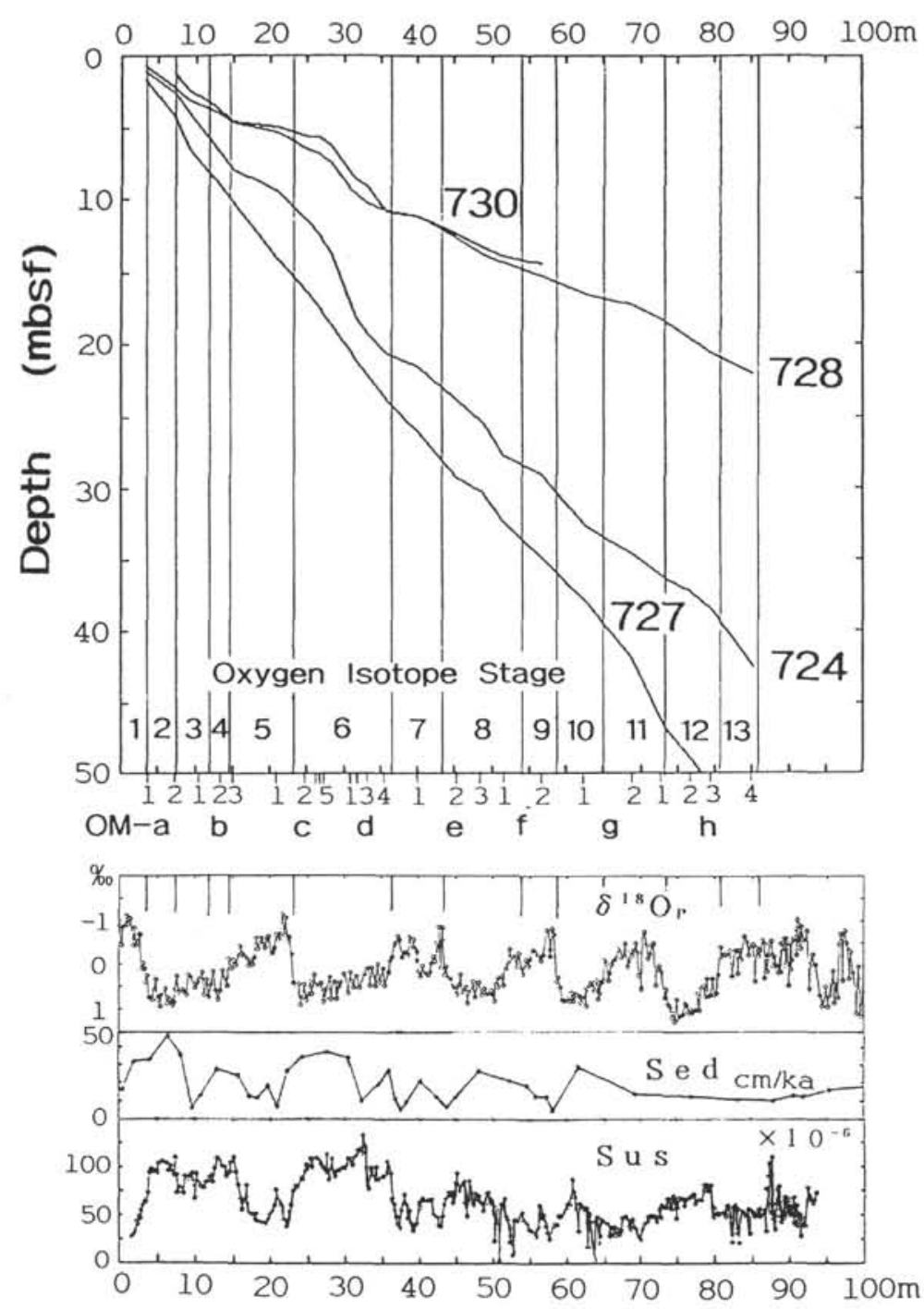

Figure 4. Depth correlation of the marker horizons in the Oman Margin and oxygen isotope stratigraphic division for Site 723. Horizontal axis is depth of the marker horizons in Site 723, vertical axis is depth in Sites 724, 727, 728, and 730. Oxygen isotope values of planktonic foraminifer in PDB $\left(\delta^{18} 0_{\mathrm{p}}\right)$, rate of sedimentation based on oxygen isotope stratigraphy (Sed), and magnetic susceptibility (Sus) in Site 723 (Niitsuma et al., this volume) are also shown. 


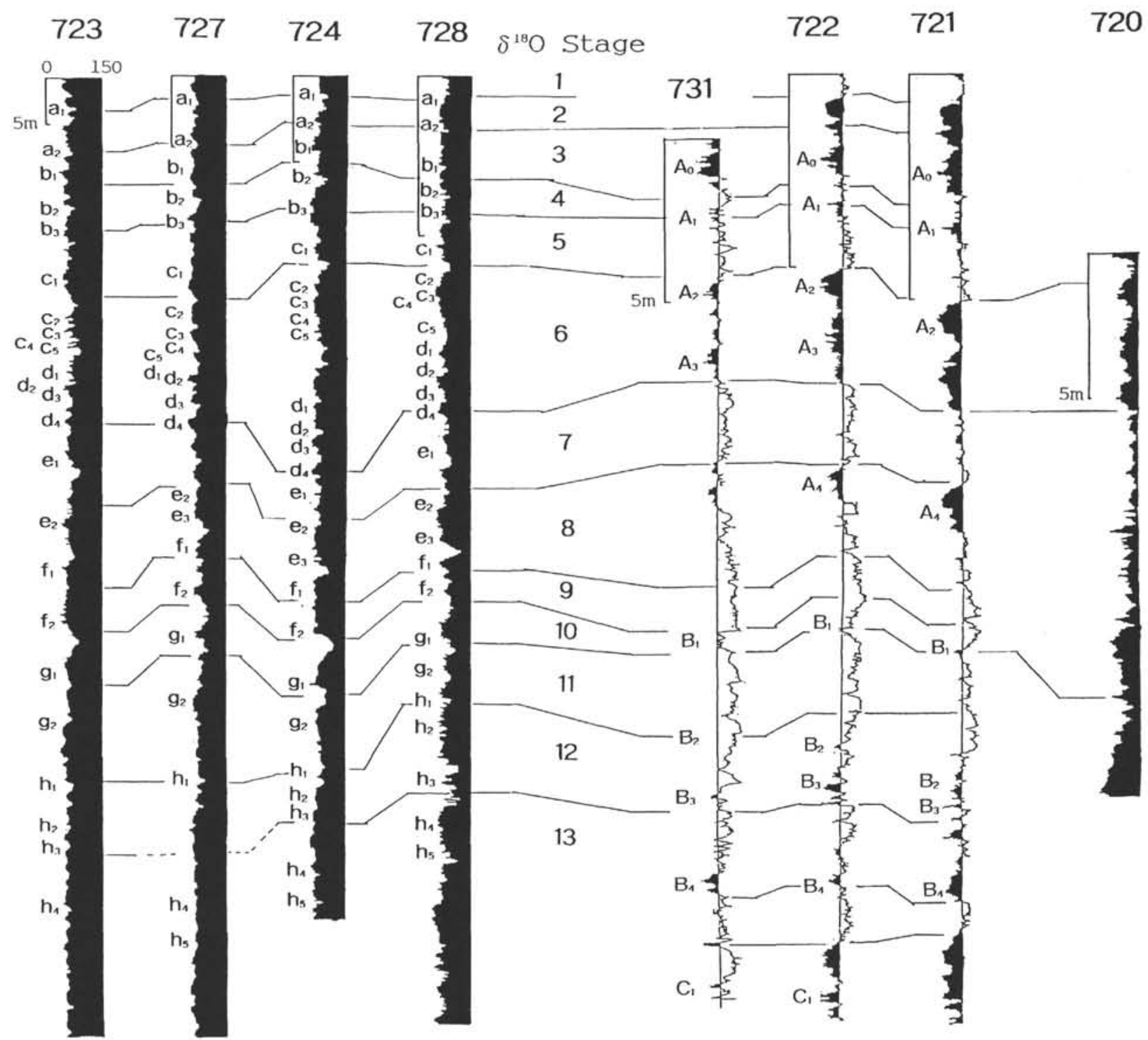

Figure 5. Comparison of color density pattern, marker horizons, and oxygen isotope stratigraphic stages in the Leg 117 sites for the last $800 \mathrm{k} . \mathrm{y}$. Vertical scale of graph for each site is enlarged or reduced for the comparison; Bar of the top-left of each graph represents $5 \mathrm{~m}$ of depth; Horizontal axis indicates the color density, measured with video-densitometer in 256 gradations: real black and real white on black and white photograph are counted as 30 and 220 of the gradation, respectively; vertical line for each site represents 150 of the gradation. 